This is an electronic reprint of the original article. This reprint may differ from the original in pagination and typographic detail.

Author(s): Lasri, Jamal; Pedras, Bruno; Haukka, Matti; Berberan-Santos, Mário N.

Title: $\quad \operatorname{Pt}(\mathrm{II})$ and $\mathrm{Pd}(\mathrm{II})$-assisted coupling of nitriles and 1,3-diiminoisoindoline : Synthesis and luminescence properties of (1,3,5,7,9-pentaazanona-1,3,6,8-tetraenato) $\mathrm{Pt}(\mathrm{II})$ and $\mathrm{Pd}(\mathrm{II})$ complexes

Year: $\quad 2017$

Version:

Please cite the original version:

Lasri, J., Pedras, B., Haukka, M., \& Berberan-Santos, M. N. (2017). Pt(II) and Pd(II)assisted coupling of nitriles and 1,3-diiminoisoindoline : Synthesis and luminescence properties of (1,3,5,7,9-pentaazanona-1,3,6,8-tetraenato) Pt(II) and $\mathrm{Pd}(\mathrm{II})$ complexes. Polyhedron, 133, 195-202. https://doi.org/10.1016/j.poly.2017.05.036

All material supplied via JYX is protected by copyright and other intellectual property rights, and duplication or sale of all or part of any of the repository collections is not permitted, except that material may be duplicated by you for your research use or educational purposes in electronic or print form. You must obtain permission for any other use. Electronic or print copies may not be offered, whether for sale or otherwise to anyone who is not an authorised user. 


\section{Accepted Manuscript}

$\mathrm{Pt}(\mathrm{II})$ and $\mathrm{Pd}(\mathrm{II})$-assisted coupling of nitriles and 1,3-diiminoisoindoline: Synthesis and luminescence properties of (1,3,5,7,9-pentaazanona-1,3,6,8-tetraenato)Pt(II) and Pd(II) complexes

Jamal Lasri, Bruno Pedras, Matti Haukka, Mário N. Berberan-Santos

PII: S0277-5387(17)30384-4

DOI: http://dx.doi.org/10.1016/j.poly.2017.05.036

Reference: POLY 12655

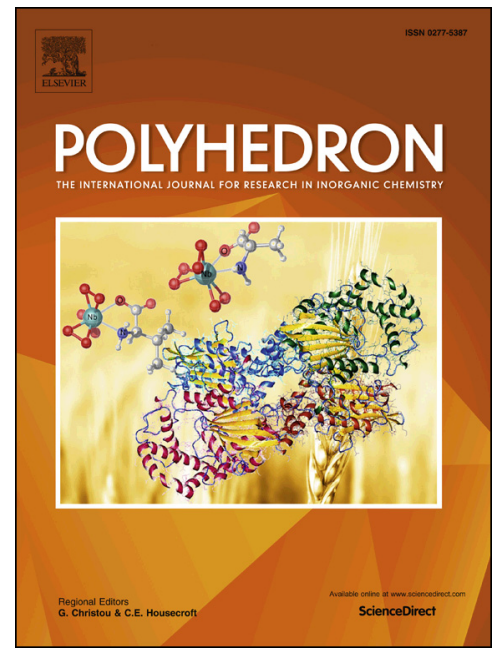

To appear in:

Polyhedron

Received Date:

24 January 2017

Revised Date:

18 May 2017

Accepted Date:

20 May 2017

Please cite this article as: J. Lasri, B. Pedras, M. Haukka, M.N. Berberan-Santos, Pt(II) and Pd(II)-assisted coupling of nitriles and 1,3-diiminoisoindoline: Synthesis and luminescence properties of (1,3,5,7,9-pentaazanona-1,3,6,8tetraenato)Pt(II) and Pd(II) complexes, Polyhedron (2017), doi: http://dx.doi.org/10.1016/j.poly.2017.05.036

This is a PDF file of an unedited manuscript that has been accepted for publication. As a service to our customers we are providing this early version of the manuscript. The manuscript will undergo copyediting, typesetting, and review of the resulting proof before it is published in its final form. Please note that during the production process errors may be discovered which could affect the content, and all legal disclaimers that apply to the journal pertain. 


\title{
Pt(II) and Pd(II)-assisted coupling of nitriles and 1,3-diiminoisoindoline: Synthesis and luminescence properties of (1,3,5,7,9-pentaazanona-1,3,6,8-tetraenato)Pt(II) and Pd(II) complexes
}

\author{
Jamal Lasri $^{\mathrm{a} * *}$, Bruno Pedras, ${ }^{\mathrm{b}}$ Matti Haukka ${ }^{\mathrm{c}}$ and Mário N. Berberan-Santos ${ }^{\mathrm{b}}$ \\ ${ }^{a}$ Department of Chemistry, Rabigh College of Science and Arts, P.O. Box 344, King Abdulaziz
} University, Jeddah, Saudi Arabia

${ }^{b}$ Centro de Química-Física Molecular, Instituto Superior Técnico, Universidade de Lisboa, Avenida Rovisco Pais, 1049-001 Lisboa, Portugal

${ }^{c}$ University of Jyväskylä, Department of Chemistry, University of Jyväskylä, P.O. Box 35, FI-40014, Finland

* Corresponding author. Tel.: +966 560247664.

E-mail address: jlasri@kau.edu.sa (J. Lasri).

\section{ABSTRACT}

Treatment of trans-[ $\left.\mathrm{PtCl}_{2}(\mathrm{NCR})_{2}\right] 1\left(\mathrm{R}=\mathrm{Me}(\mathbf{1 a}), \mathrm{Et}(\mathbf{1 b}), o-\mathrm{ClC}_{6} \mathrm{H}_{4}(\mathbf{1 c}), p-\mathrm{ClC}_{6} \mathrm{H}_{4}(\mathbf{1 d}), p\right.$ $(\mathrm{HC}=\mathrm{O}) \mathrm{C}_{6} \mathrm{H}_{4}(\mathbf{1 e}), p-\mathrm{O}_{2} \mathrm{NC}_{6} \mathrm{H}_{4} \mathrm{CH}_{2}$ (1) $)$ with 1,3-diiminoisoindoline $\mathrm{HN}=\mathrm{CC}_{6} \mathrm{H}_{4} \mathrm{C}(\underline{\mathrm{NH}})=\mathrm{NH} 2$ gives access to the corresponding (1,3,5,7,9-pentaazanona-1,3,6,8-tetraenato) $\mathrm{Pt}(\mathrm{II})$ complexes $\left[\mathrm{PtCl}\left\{\underline{\mathrm{NH}}=\mathrm{C}(\mathrm{R}) \mathrm{N}=\mathrm{C}\left(\mathrm{C}_{6} \mathrm{H}_{4}\right) \underline{\mathrm{NC}}=\mathrm{NC}(\mathrm{R})=\underline{\mathrm{NH}}\right\}\right]$ 3a-f, in good yields $(65-70 \%)$. The reaction of trans$\left[\mathrm{PdCl}_{2}(\mathrm{NCMe})_{2}\right]$ 4a with 2 furnishes (1,3,5,7,9-pentaazanona-1,3,6,8-tetraenato) $\mathrm{Pd}(\mathrm{II})$ complex $\left[\mathrm{PdCl}\left\{\underline{\mathrm{NH}}=\mathrm{C}(\mathrm{Me}) \mathrm{N}=\mathrm{C}\left(\mathrm{C}_{6} \mathrm{H}_{4}\right) \underline{\mathrm{NC}}=\mathrm{NC}(\mathrm{Me})=\underline{\mathrm{NH}}\right\}\right] \mathbf{5 a}$, in good yield $(65 \%)$. However, the reaction of trans- $\left[\mathrm{PdCl}_{2}(\mathrm{NCR})_{2}\right] 4\left(\mathrm{R}=\mathrm{Ph}(\mathbf{4 b}), p-\mathrm{MeC}_{6} \mathrm{H}_{4} \mathrm{CH}_{2}(\mathbf{4 c}), p-(\mathrm{HC}=\mathrm{O}) \mathrm{C}_{6} \mathrm{H}_{4}(\mathbf{4 d}), p-\mathrm{O}_{2} \mathrm{NC}_{6} \mathrm{H}_{4} \mathrm{CH}_{2}(\mathbf{4 e})\right)$ with 2 gives a number of unidentified products. The compounds 3a-f and 5a were characterized by IR, ${ }^{1} \mathrm{H},{ }^{13} \mathrm{C}$ and DEPT-135 NMR spectroscopies, elemental analyses and, in the case of the Pt(II) complex $\left[\mathrm{PtCl}\left\{\underline{\mathrm{NH}}=\mathrm{C}(\mathrm{Me}) \mathrm{N}=\mathrm{C}\left(\mathrm{C}_{6} \mathrm{H}_{4}\right) \underline{\mathrm{NC}}=\mathrm{NC}(\mathrm{Me})=\underline{\mathrm{NH}}\right\}\right]$ 3a, also by X-ray diffraction analysis. Compounds 3a and $\mathbf{3 b}$ were also characterized by UV-vis absorption and luminescence emission spectroscopies. Emission quantum yields of $c a .3 \times 10^{-3}$ were obtained in dichloromethane solution, and luminescence lifetimes are in the order of the tens of nanoseconds. Both compounds also exhibited luminescence in solid state (polystyrene matrix), with luminescence lifetimes in the order of hundreds of nanoseconds.

Keywords: Metal-assisted additions; Nitriles; 1,3-Diiminoisoindoline; Pentaazanonatetraene complexes; Luminescence. 


\section{Introduction}

Several methods to prepare coordination compounds by nucleophilic additions to nitrile ligands have been developed during the last two decades [1]. Various types of nucleophiles [2,3] or 1,3-dipoles [4] have been used for the preparation of compounds containing $\mathrm{C}-\mathrm{N}$ and/or $\mathrm{C}-\mathrm{O}$ bonds. 1,3Diiminoisoindoline has been used for the synthesis of pthalocyanines [5] and hemiporphyrazine [6], which have a wide range of industrial applications. The iminoisoindoline-1-one, bearing a nucleophilic $s p^{2}$-imino group, has been used as a nucleophile in reaction with various metal-bound isonitriles and nitriles to furnish iminocarbene or triazapentadienato complexes, respectively [7]. However, 1,3diiminoisoindoline contains two $s p^{2}$-nitrogen centres which can use both imine moieties for additions to metal-coordinated nitriles, thus furnishing symmetrical triazapentadienate complexes. In contrast to $\beta$ diimines, ligands such as triazapentadienes have one extra $N$ donor site, and DFT [8] show that they have a greater capacity of coordination to metal ions than $\beta$-diimines. Nevertheless, the coordination chemistry of triazapendiene species is less reported, due to the instability of triazapentadiene complexes, particularly the unsubstituted ones [9]. A single-pot synthesis with electron-deficient nitriles has been used to synthetize triazapentadiene complexes [10]. $\mathrm{Ni}$ (II)-complexes bearing imidoylamidine ligands have been prepared using oximes and nitriles in the presence of $\mathrm{Ni}(\mathrm{II})$ ions [11], and this methodology has been used to synthetize a variety of (1,3,5-triazapentadienato)Pd(II) complexes [12]. Recently, we have also reported the synthesis of (alkoxy-1,3,5-triazapentadienato) $\mathrm{Cu}(\mathrm{II})$ complexes using a template synthesis [13].

On the other hand, Pt(II)-based imidoylamidinate compounds are emissive both in solid state and in solution, at room temperature. UV-visible and luminescence spectroscopies indicate that the lowest excited state of these complexes is ${ }^{3}$ MLCT or ${ }^{3}$ IL with significant MLCT character, with emission lifetimes of a few $\mu$ s [14].

In continuation of our research program on the additions to metal-bound nitriles [15], we decided to extend the addition of a $s p^{2}$-nitrogen nucleophile, 1,3-diiminoisoindoline $\mathrm{HN}=\underline{\mathrm{C}} \mathrm{C}_{6} \mathrm{H}_{4} \mathrm{C}(\underline{\mathrm{NH}})=\mathrm{NH} 2$, i) to various $\mathrm{Pt}(\mathrm{II})$-bound nitriles trans-[ $\left.\mathrm{PtCl}_{2}(\mathrm{NCR})_{2}\right] \mathbf{1}\left(\mathrm{R}=\mathrm{Me}(\mathbf{1 a}), \mathrm{Et}(\mathbf{1 b}), o-\mathrm{ClC}_{6} \mathrm{H}_{4}(\mathbf{1 c}), p-\mathrm{ClC}_{6} \mathrm{H}_{4}\right.$ (1d), $p$ - $(\mathrm{HC}=\mathrm{O}) \mathrm{C}_{6} \mathrm{H}_{4}(\mathbf{1 e}), p-\mathrm{O}_{2} \mathrm{NC}_{6} \mathrm{H}_{4} \mathrm{CH}_{2}$ (1f)); and ii) to another type of metal complex such as $\mathrm{Pd}(\mathrm{II})$-bound acetonitrile trans-[ $\left.\mathrm{PdCl}_{2}(\mathrm{NCMe})_{2}\right] \mathbf{4 a}$; and also iii) to investigate the UV-vis absorption and the luminescence emission spectra of some of those complexes. We have thus observed the formation of (1,3,5,7,9-pentaazanona-1,3,6,8-tetraenato) $\mathrm{Pt}(\mathrm{II})$ and $\mathrm{Pd}(\mathrm{II})$ complexes, 3a-f and 5a, respectively. The photophysical characterization of compounds $\mathbf{3 a}$ and $\mathbf{3 b}$ permitted the assessment of 
parameters which, by correlation with other Pt(II) emissive complexes [14], enabled further insight into their electronic structure, namely in terms of optical transitions.

\section{Experimental section}

\subsection{General methods}

${ }^{1} \mathrm{H},{ }^{13} \mathrm{C}$ and DEPT-135 NMR spectra (in $\mathrm{CDCl}_{3}$ or DMSO- $d_{6}$ ) were measured on Bruker Avance III HD $600 \mathrm{MHz}$ (Ascend ${ }^{\mathrm{TM}}$ Magnet) spectrometer at ambient temperature. ${ }^{1} \mathrm{H},{ }^{13} \mathrm{C}$ and DEPT-135 chemical shifts $(\delta)$ are expressed in ppm relative to TMS. Infrared spectra $\left(400-4000 \mathrm{~cm}^{-1}\right)$ were recorded on an Alpha Bruker FT-IR instrument in KBr pellets. C, $\mathrm{H}$ and $\mathrm{N}$ elemental analyses were carried out by the Microanalytical Service of the King Abdulaziz University.

The solvents used for photophysical characterization were all of spectroscopic grade. Dichloromethane (99.5\% for spectroscopy, Acros Organics), chloroform ( $\geq 99.8 \%$ ACS spectrometric grade, SigmaAldrich), ethanol (95\%, UV HPLC spectroscopic, Sigma-Aldrich). Deionized water was obtained from a Millipore system Milli-Q $\geq 18 \mathrm{M} \Omega \mathrm{cm}$. Polystyrene beads (average $\mathrm{M}_{\mathrm{w}}$ 35000, Sigma-Aldrich) was used to prepare solid films of compounds $\mathbf{3 a}$ and $\mathbf{3 b}$, by dissolving $1 \mathrm{mg}$ of the respective compound in a solution of $c a .80 \mathrm{mg}$ of polystyrene in $1 \mathrm{~mL}$ chloroform, which was subsequently deposited over a quartz plate, allowing the solvent to evaporate. Luminescence quantum yields were calculated by using $\mathrm{Ru}(\mathrm{bpy})_{3}$ as reference quantum yield standard for compounds $\mathbf{3 a}$ and $\mathbf{3 b}$. The electronic absorption spectra were recorded using a Jasco V-660 spectrophotometer. Fluorescence measurements were carried out in a Horiba-Jobin Yvon Fluorolog-3 spectrofluorimeter. All spectra were recorded with samples in 1 $\mathrm{cm}$ optical path length quartz cells, except for the solid films, where the film was placed directly in the optical path, in a $45^{\circ}$ angle between the excitation source and the detector. Luminescence lifetime measurements were performed using the single-photon timing method with laser excitation and microchannel plate detection, with the set-up already described [16]. Excitation wavelength used was $335 \mathrm{~nm}$ for solution and $304 \mathrm{~nm}$ for solid state and the emission wavelength was $605 \mathrm{~nm}$. The timescale

was $438.2 \mathrm{ps} / \mathrm{channel}$ for solution measurements, $679.2 \mathrm{ps} / \mathrm{channel}$ for solid state $\mathbf{3 a}$ and 786.7 ps/channel for solid state $\mathbf{3 b}$.

2.2. Preparation of the nitrile Pt(II) complexes trans-[PtCl $\left.l_{2}(\mathrm{NCR})_{2}\right] \mathbf{1}(R=\mathrm{Me}(\mathbf{l a})$, Et $(\boldsymbol{1} \boldsymbol{b}), o-$ $\left.\mathrm{ClC}_{6} \mathrm{H}_{4}(\mathbf{l c}), \mathrm{p}-\mathrm{ClC}_{6} \mathrm{H}_{4}(\mathbf{l d}), \mathrm{p}-(\mathrm{HC}=\mathrm{O}) \mathrm{C}_{6} \mathrm{H}_{4}(\mathbf{l e}), \mathrm{p}-\mathrm{O}_{2} \mathrm{NC}_{6} \mathrm{H}_{4} \mathrm{CH}_{2}(\mathbf{l f})\right)$ and their reactions with $1,3-$ diiminoisoindoline 2 


\subsubsection{Reaction of $\mathrm{PtCl}_{2}$ with liquid nitriles}

$\mathrm{PtCl}_{2}$ (200 mg, $0.752 \mathrm{mmol}$ ) was added at room temperature to acetonitrile or propionitrile ( $5 \mathrm{~mL}$ ), and the mixture was heated under stirring at $70{ }^{\circ} \mathrm{C}$ for $8 \mathrm{~h}$. During the course of the reaction, the green gray $\mathrm{PtCl}_{2}$ powder dissolved, forming a homogeneous light yellow solution. The reaction mixture was then dried in vacuo, washed with three $5 \mathrm{~mL}$ portions of diethyl ether and dried under air to give trans$\left[\mathrm{PtCl}_{2}(\mathrm{NCMe})_{2}\right]$ 1a or trans-[ $\left.\mathrm{PtCl}_{2}(\mathrm{NCEt})_{2}\right] \mathbf{1 b}$, respectively, in excellent yields (ca. $\left.90 \%\right)$.

\subsubsection{Reaction of $\mathrm{PtCl}_{2}$ with solid nitriles}

$\mathrm{PtCl}_{2}(200 \mathrm{mg}, 0.752 \mathrm{mmol}$ ) was added at room temperature to $o$-chlorobenzonitrile $(1034 \mathrm{mg}, 7.52$ mmol), $p$-chlorobenzonitrile (1034 mg, $7.52 \mathrm{mmol}$ ), p-cyanobenzaldehyde (986 mg, $7.52 \mathrm{mmol}$ ) or $p$ nitrophenylacetonitrile (1218 $\mathrm{mg}, 7.52 \mathrm{mmol})$, and the mixture was heated at the melting point of each nitrile $\left(46,93,102\right.$ or $115^{\circ} \mathrm{C}$, respectively) in a sealed glass vial for $12 \mathrm{~h}$. Also in this case, during the course of the reaction, the green gray $\mathrm{PtCl}_{2}$ powder dissolved in the melted nitrile, forming a homogeneous light yellow solution. The excess of nitrile was then removed by washing the reaction mixture with three $10 \mathrm{~mL}$ portions of diethyl ether or chloroform (depending on the solubility of the nitrile used $)$ and dried under air to give trans- $\left[\mathrm{PtCl}_{2}\left(\mathrm{NC}\left(o-\mathrm{ClC}_{6} \mathrm{H}_{4}\right)\right)_{2}\right] \mathbf{1 c}$, trans- $\left[\mathrm{PtCl}_{2}\left(\mathrm{NC}\left(p-\mathrm{ClC}_{6} \mathrm{H}_{4}\right)\right)_{2}\right]$ 1d, trans- $\left[\mathrm{PtCl}_{2}\left(\mathrm{NC}\left(p-(\mathrm{HC}=\mathrm{O}) \mathrm{C}_{6} \mathrm{H}_{4}\right)\right)_{2}\right]$ 1e or trans- $\left[\mathrm{PtCl}_{2}\left(\mathrm{NC}\left(p-\mathrm{O}_{2} \mathrm{NC}_{6} \mathrm{H}_{4} \mathrm{CH}_{2}\right)\right)_{2}\right]$ 1f, respectively, in excellent yields ( $c a .87 \%)$.

To confirm the formation of those Pt(II) complexes, the new compounds e.g. 1c and 1e were characterized by ${ }^{1} \mathrm{H},{ }^{13} \mathrm{C}$ and DEPT-135 NMR spectroscopy, then the NMR data were compared with their respective starting materials ( $o$-chlorobenzonitrile and $p$-cyanobenzaldehyde).

$o$-Chlorobenzonitrile $\mathrm{N} \equiv \mathrm{C}\left(o-\mathrm{ClC}_{6} \mathrm{H}_{4}\right)$

${ }^{1} \mathrm{H}$ NMR $\left(\mathrm{CDCl}_{3}\right), \delta: 7.40\left(\mathrm{t}, J_{\mathrm{HH}} 7.5 \mathrm{~Hz}, 1 \mathrm{H}, \mathrm{C} H_{\text {aromatic }}\right), 7.53-7.58$ (m, 2H, $\left.\mathrm{CH}_{\text {aromatic }}\right), 7.69$ (d, $J_{\mathrm{HH}} 7.7$

$\left.\mathrm{Hz}, 1 \mathrm{H}, \mathrm{CH}_{\text {aromatic }}\right) .{ }^{13} \mathrm{C} \mathrm{NMR}\left(\mathrm{CDCl}_{3}\right), \delta: 113.4(C \equiv \mathrm{N}), 115.9\left(\mathrm{Cl}-C_{\text {aromatic }}\right), 127.1,130.1,133.9,134.0$, 136.9 ( $\left.\mathrm{C}_{\text {aromatic }}\right)$. DEPT-135 NMR $\left(\mathrm{CDCl}_{3}\right), \delta: 127.1,130.1,133.9,134.0\left(\mathrm{CH}_{\text {aromatic }}\right)$.

Trans- $\left[\mathrm{PtCl}_{2}\left(\mathrm{NC}\left(o-\mathrm{ClC}_{6} \mathrm{H}_{4}\right)\right)_{2}\right](\mathbf{1 c})$

Yield: 87\%. ${ }^{1} \mathrm{H}$ NMR $\left(\mathrm{CDCl}_{3}\right), \delta: 7.49\left(\mathrm{t}, J_{\mathrm{HH}} 7.7 \mathrm{~Hz}, 2 \mathrm{H}, \mathrm{CH}_{\text {aromatic }}\right), 7.60\left(\mathrm{~d}, J_{\mathrm{HH}} 8.2 \mathrm{~Hz}, 2 \mathrm{H}\right.$, $\left.\mathrm{CH}_{\text {aromatic }}\right), 7.70\left(\mathrm{t}, J_{\mathrm{HH}} 7.7 \mathrm{~Hz}, 2 \mathrm{H}, \mathrm{CH}_{\text {aromatic }}\right), 7.80\left(\mathrm{~d}, J_{\mathrm{HH}} 7.8 \mathrm{~Hz}, 2 \mathrm{H}, \mathrm{CH}_{\text {aromatic }}\right) .{ }^{13} \mathrm{C} \mathrm{NMR}\left(\mathrm{CDCl}_{3}\right), \delta$ : 
$110.5\left(\mathrm{Cl}-C_{\text {aromatic }}\right), 114.1(C \equiv \mathrm{N}), 127.4,130.5,135.5,136.3,138.9$ (C $\left.\mathrm{C}_{\text {aromatic }}\right)$. DEPT-135 NMR $\left(\mathrm{CDCl}_{3}\right), \delta: 127.4,130.5,135.5,136.3\left(\mathrm{CH}_{\text {aromatic }}\right)$.

$p$-Cyanobenzaldehyde $\mathrm{N} \equiv \mathrm{C}\left(p\right.$ - $\left.(\mathrm{HC}=\mathrm{O}) \mathrm{C}_{6} \mathrm{H}_{4}\right)$

${ }^{1} \mathrm{H}$ NMR $\left(\mathrm{CDCl}_{3}\right), \delta: 7.86\left(\mathrm{~d}, J_{\mathrm{HH}} 8.3 \mathrm{~Hz}, 2 \mathrm{H}, \mathrm{CH}_{\text {aromatic }}\right), 8.00\left(\mathrm{~d}, J_{\mathrm{HH}} 8.3 \mathrm{~Hz}, 2 \mathrm{H}, \mathrm{C} H_{\text {aromatic }}\right), 10.10$ (s, $1 \mathrm{H}, H \mathrm{C}=\mathrm{O}) .{ }^{13} \mathrm{C} \mathrm{NMR}\left(\mathrm{CDCl}_{3}\right), \delta: 117.6$ and $117.7(C \equiv \mathrm{N}), 129.9,132.9,138.7\left(\mathrm{C}_{\text {aromatic }}\right), 190.6$ $(\mathrm{HC}=\mathrm{O})$. DEPT-135 NMR $\left(\mathrm{CDCl}_{3}\right), \delta: 129.9,132.9\left(\mathrm{CH}_{\text {aromatic }}\right), 190.6(\mathrm{HC}=\mathrm{O})$.

\section{Trans- $\left[\mathrm{PtCl}_{2}\left(\mathrm{NC}\left(p-(\mathrm{HC}=\mathrm{O}) \mathrm{C}_{6} \mathrm{H}_{4}\right)\right)_{2}\right](\mathbf{1 e})$}

Yield: 85\%. ${ }^{1} \mathrm{H}$ NMR $\left(\mathrm{CDCl}_{3}\right), \delta: 8.04\left(\mathrm{~d}, J_{\mathrm{HH}} 8.4 \mathrm{~Hz}, 4 \mathrm{H}, \mathrm{CH}_{\text {aromatic }}\right), 8.10$ (d, $J_{\mathrm{HH}} 8.4 \mathrm{~Hz}, 4 \mathrm{H}$, $\left.\mathrm{CH}_{\text {aromatic }}\right), 10.16(\mathrm{~s}, 2 \mathrm{H}, H \mathrm{C}=\mathrm{O}) .{ }^{13} \mathrm{C} \mathrm{NMR}\left(\mathrm{CDCl}_{3}\right), \delta: 113.9$ and $115.8(\mathrm{C} \equiv \mathrm{N}), 129.9,134.6,140.3$ $\left(\mathrm{C}_{\text {aromatic }}\right), 190.0(\mathrm{HC}=\mathrm{O})$. DEPT-135 NMR $\left(\mathrm{CDCl}_{3}\right), \delta: 129.9,134.6\left(\mathrm{CH}_{\text {aromatic }}\right), 190.0(\mathrm{HC}=\mathrm{O})$.

2.2.3. Reactions of the nitrile Pt(II) complexes trans-[PtCl $\left.l_{2}(\mathrm{NCR})_{2}\right] \mathbf{1}(\mathrm{R}=\mathrm{Me}(\mathbf{l a})$, Et (1b), o$\left.\mathrm{ClC}_{6} \mathrm{H}_{4}(\mathbf{1 c}), \mathrm{p}-\mathrm{ClC}_{6} \mathrm{H}_{4}(\mathbf{l d}), \mathrm{p}-(\mathrm{HC}=\mathrm{O}) \mathrm{C}_{6} \mathrm{H}_{4}(\mathbf{l e}), p-\mathrm{O}_{2} \mathrm{NC}_{6} \mathrm{H}_{4} \mathrm{CH}_{2}(\mathbf{l f})\right)$ with 1,3-diiminoisoindoline 2

To a solution of $\mathbf{1 a}, \mathbf{1 b}, \mathbf{1 c}, \mathbf{1 d}, \mathbf{1 e}$ or $\mathbf{1 f}(0.532 \mathrm{mmol})$ in chloroform $(5 \mathrm{~mL})$ was added at room temperature to 1,3-diiminoisoindoline $2(77.2 \mathrm{mg}, 0.532 \mathrm{mmol})$, and the mixture was refluxed for $2 \mathrm{~h}$ whereupon the solvent was removed in vacuo. The crude residue was purified by column chromatography on silica (chloroform as the eluent), followed by evaporation of the solvent in vacuo to give the final $\mathbf{3 a}, \mathbf{3 b}, \mathbf{3 c}, \mathbf{3 d}, \mathbf{3 e}$ or $\mathbf{3 f}$ products, respectively.

\section{$\left[\mathrm{PtCl}\left\{\underline{\mathrm{NH}}=\mathrm{C}(\mathrm{Me}) \mathrm{N}=\mathrm{C}\left(\mathrm{C}_{6} \mathrm{H}_{4}\right) \underline{\mathrm{NC}}=\mathrm{NC}(\mathrm{Me})=\underline{\mathrm{NH}}\right\}\right](\mathbf{3 a})$}

Yield: 67\%. IR $\left(\mathrm{cm}^{-1}\right): 3441(\mathrm{NH}), 1628(\mathrm{C}=\mathrm{N}) .{ }^{1} \mathrm{H}$ NMR $\left(\mathrm{CDCl}_{3}\right), \delta: 2.55\left(\mathrm{~s}, 6 \mathrm{H}, \mathrm{CH}_{3}\right), 7.75\left(\mathrm{dd}, J_{\mathrm{HH}}\right.$ 3.0 and $\left.5.5 \mathrm{~Hz}, 2 \mathrm{H}, \mathrm{CH}_{\text {aromatic }}\right), 8.24\left(\mathrm{dd}, J_{\mathrm{HH}} 3.0\right.$ and $\left.5.5 \mathrm{~Hz}, 2 \mathrm{H}, \mathrm{CH}_{\text {aromatic }}\right), 9.80(\mathrm{~s}, \mathrm{br}, 2 \mathrm{H}, \mathrm{NH}) .{ }^{13} \mathrm{C}$ $\operatorname{NMR}\left(\mathrm{CDCl}_{3}\right), \delta: 28.8\left(\mathrm{CH}_{3}\right), 123.2,132.2,137.9\left(C_{\text {aromatic }}\right), 153.1,159.8(C=\mathrm{N})$. DEPT-135 NMR $\left(\mathrm{CDCl}_{3}\right), \delta: 28.8\left(\mathrm{CH}_{3}\right), 123.2,132.2\left(\mathrm{CH}_{\text {aromatic }}\right)$. Anal. Calcd for $\mathrm{C}_{12} \mathrm{H}_{12} \mathrm{~N}_{5} \mathrm{ClPt}(456.79): \mathrm{C}, 31.55 ; \mathrm{H}$, 2.65; N, 15.33. Found: C, 31.67; H, 2.74; N, 15.28.

\section{$\left[\mathrm{PtCl}\left\{\underline{\mathrm{NH}}=\mathrm{C}(\mathrm{Et}) \mathrm{N}=\mathrm{C}\left(\mathrm{C}_{6} \mathrm{H}_{4}\right) \underline{\mathrm{NC}}=\mathrm{NC}(\mathrm{Et})=\underline{\mathrm{NH}}\right\}\right](\mathbf{3 b})[15]$}

Yield: 65\%. IR $\left(\mathrm{cm}^{-1}\right): 3443(\mathrm{NH}), 1615(\mathrm{C}=\mathrm{N}) .{ }^{1} \mathrm{H}$ NMR $\left(\mathrm{CDCl}_{3}\right), \delta: 1.47\left(\mathrm{t}, J_{\mathrm{HH}} 7.5 \mathrm{~Hz}, 6 \mathrm{H}, \mathrm{CH}_{3} \mathrm{CH}_{2}\right)$, $2.82\left(\mathrm{q}, J_{\mathrm{HH}} 7.5 \mathrm{~Hz}, 4 \mathrm{H}, \mathrm{CH}_{3} \mathrm{CH}_{2}\right), 7.75\left(\mathrm{dd}, J_{\mathrm{HH}} 3.0\right.$ and $\left.5.4 \mathrm{~Hz}, 2 \mathrm{H}, \mathrm{CH}_{\text {aromatic }}\right), 8.26\left(\mathrm{dd}, J_{\mathrm{HH}} 3.0\right.$ and $5.4 \mathrm{~Hz}, 2 \mathrm{H}, \mathrm{CH}$ aromatic $), 9.77(\mathrm{~s}, \mathrm{br}, 2 \mathrm{H}, \mathrm{NH}) .{ }^{13} \mathrm{C} \mathrm{NMR}\left(\mathrm{CDCl}_{3}\right), \delta: 11.0\left(\mathrm{CH}_{3}\right), 35.1\left(\mathrm{CH}_{2}\right), 123.2$, 
132.1, $137.9\left(\mathrm{C}_{\text {aromatic }}\right), 153.2$ and $163.8(C=\mathrm{N})$. DEPT-135 NMR $\left(\mathrm{CDCl}_{3}\right), \delta: 11.0\left(\mathrm{CH}_{3}\right), 35.1\left(C \mathrm{H}_{2}\right)$, 123.2, $132.1\left(\mathrm{CH}_{\text {aromatic }}\right)$. Calcd for $\mathrm{C}_{14} \mathrm{H}_{16} \mathrm{~N}_{5} \mathrm{ClPt}$ (484.84): C, 34.68; H, 3.33; N, 14.44. Found: $\mathrm{C}$, $34.74 ; \mathrm{H}, 3.25 ; \mathrm{N}, 14.33$.

$\left[\mathrm{PtCl}\left\{\underline{\mathrm{NH}}=\mathrm{C}\left(o-\mathrm{ClC}_{6} \mathrm{H}_{4}\right) \mathrm{N}=\mathrm{C}\left(\mathrm{C}_{6} \mathrm{H}_{4}\right) \underline{\mathrm{NC}}=\mathrm{NC}\left(o-\mathrm{ClC}_{6} \mathrm{H}_{4}\right)=\underline{\mathrm{NH}}\right\}\right](\mathbf{3 c})$

Yield: 68\%. IR $\left(\mathrm{cm}^{-1}\right): 2926(\mathrm{NH}), 1678(\mathrm{C}=\mathrm{N}) .{ }^{1} \mathrm{H} \mathrm{NMR}\left(\mathrm{CDCl}_{3}\right), \delta: 7.48\left(\mathrm{t}, J_{\mathrm{HH}} 7.7 \mathrm{~Hz}, 2 \mathrm{H}\right.$, $\left.\mathrm{CH}_{\text {aromatic }}\right), 7.53-7.61\left(\mathrm{~m}, 4 \mathrm{H}, \mathrm{CH}_{\text {aromatic }}\right), 7.70\left(\mathrm{t}, J_{\mathrm{HH}} 7.7 \mathrm{~Hz}, 2 \mathrm{H}, \mathrm{CH}_{\text {aromatic }}\right), 7.80\left(\mathrm{~m}, 2 \mathrm{H}, \mathrm{C} H_{\text {aromatic }}\right)$, $8.04\left(\mathrm{~d}, J_{\mathrm{HH}} 7.6 \mathrm{~Hz}, 1 \mathrm{H}, \mathrm{CH}_{\text {aromatic }}\right), 8.36\left(\mathrm{dd}, J_{\mathrm{HH}} 3.0\right.$ and $\left.5.5 \mathrm{~Hz}, 1 \mathrm{H}, \mathrm{CH}_{\text {aromatic }}\right), 10.80(\mathrm{~s}, \mathrm{br}, 2 \mathrm{H}, \mathrm{NH})$. ${ }^{13} \mathrm{C} \mathrm{NMR}\left(\mathrm{CDCl}_{3}\right), \delta: 110.5$ (Cl- $\left.C_{\text {aromatic }}\right), 123.6,127.3,127.4,130.5,130.7,131.0,131.6,132.4,135.5$, 136.3, $138.9\left(\mathrm{C}_{\text {aromatic }}\right), 153.7$ and $155.9(C=\mathrm{N})$. DEPT-135 NMR $\left(\mathrm{CDCl}_{3}\right), \delta$ : 123.6, 127.3, 127.4, 130.5, 130.7, 131.0, 131.6, 132.4, 135.5, 136.3 ( $\left.\mathrm{CH}_{\text {aromatic }}\right)$. Anal. Calcd for $\mathrm{C}_{22} \mathrm{H}_{14} \mathrm{~N}_{5} \mathrm{Cl}_{3} \mathrm{Pt}$ (649.82): C, 40.66; H, 2.17; N, 10.78. Found: C, 40.78; H, 2.55; N, 10.21.

\section{$\left[\mathrm{PtCl}\left\{\underline{\mathrm{NH}}=\mathrm{C}\left(p-\mathrm{ClC}_{6} \mathrm{H}_{4}\right) \mathrm{N}=\mathrm{C}\left(\mathrm{C}_{6} \mathrm{H}_{4}\right) \underline{\mathrm{NC}}=\mathrm{NC}\left(p-\mathrm{ClC}_{6} \mathrm{H}_{4}\right)=\underline{\mathrm{NH}}\right\}\right](\mathbf{3 d})$}

Yield: 69\%. IR $\left(\mathrm{cm}^{-1}\right): 3196(\mathrm{NH}), 1677(\mathrm{C}=\mathrm{N}) .{ }^{1} \mathrm{H} \mathrm{NMR}\left(\mathrm{CDCl}_{3}\right), \delta: 7.58\left(\mathrm{~d}, J_{\mathrm{HH}} 8.6 \mathrm{~Hz}, 6 \mathrm{H}\right.$, $\left.\mathrm{CH}_{\text {aromatic }}\right), 7.77\left(\mathrm{~d}, J_{\mathrm{HH}} 8.6 \mathrm{~Hz}, 4 \mathrm{H}, \mathrm{CH}_{\text {aromatic }}\right), 8.24\left(\mathrm{~d}, J_{\mathrm{HH}} 8.6 \mathrm{~Hz}, 1 \mathrm{H}, \mathrm{CH}_{\text {aromatic }}\right), 8.36$ (dd, $J_{\mathrm{HH}} 3.0$ and $\left.5.4 \mathrm{~Hz}, 1 \mathrm{H}, \mathrm{CH}_{\text {aromatic }}\right), 10.36(\mathrm{~s}, \mathrm{br}, 2 \mathrm{H}, \mathrm{NH}) .{ }^{13} \mathrm{C} \mathrm{NMR}\left(\mathrm{CDCl}_{3}\right), \delta: 107.2\left(\mathrm{Cl}-C_{\text {aromatic }}\right), 123.5,128.8$, $129.5,130.2,132.4,134.9,142.7\left(\mathrm{C}_{\text {aromatic }}\right), 153.9$ and $155.3(C=\mathrm{N})$. DEPT-135 NMR $\left(\mathrm{CDCl}_{3}\right), \delta$ : 123.5, 128.8, 129.5, 130.2, 132.4, $134.9\left(\mathrm{CH}_{\text {aromatic }}\right)$. Anal. Calcd for $\mathrm{C}_{22} \mathrm{H}_{14} \mathrm{~N}_{5} \mathrm{Cl}_{3} \mathrm{Pt}$ (649.82): C, 40.66; H, 2.17; N, 10.78. Found: C, 40.53; H, 2.45; N, 10.61 .

\section{$\left[\mathrm{PtCl}\left\{\underline{\mathrm{NH}}=\mathrm{C}\left(p-(\mathrm{HC}=\mathrm{O}) \mathrm{C}_{6} \mathrm{H}_{4}\right) \mathrm{N}=\mathrm{C}\left(\mathrm{C}_{6} \mathrm{H}_{4}\right) \underline{\mathrm{NC}}=\mathrm{NC}\left(p-(\mathrm{HC}=\mathrm{O}) \mathrm{C}_{6} \mathrm{H}_{4}\right)=\underline{\mathrm{NH}}\right\}\right](\mathbf{3 e})$}

Yield: 67\%. IR $\left(\mathrm{cm}^{-1}\right): 3424(\mathrm{NH}), 1607(\mathrm{C}=\mathrm{N}), 1702(\mathrm{C}=\mathrm{O}) .{ }^{1} \mathrm{H}$ NMR $\left(\mathrm{CDCl}_{3}\right), \delta: 7.87\left(\mathrm{dd}, J_{\mathrm{HH}} 3.0\right.$ and $\left.5.4 \mathrm{~Hz}, 2 \mathrm{H}, \mathrm{CH}_{\text {aromatic }}\right), 8.11\left(\mathrm{~d}, J_{\mathrm{HH}} 8.4 \mathrm{~Hz}, 4 \mathrm{H}, \mathrm{CH}_{\text {aromatic }}\right), 8.44\left(\mathrm{dd}, J_{\mathrm{HH}} 3.0\right.$ and $5.4 \mathrm{~Hz}, 2 \mathrm{H}$, $\left.\mathrm{CH}_{\text {aromatic }}\right), 8.48\left(\mathrm{~d}, J_{\mathrm{HH}} 8.4 \mathrm{~Hz}, 4 \mathrm{H}, \mathrm{CH}_{\text {aromatic }}\right), 10.20(\mathrm{~s}, 2 \mathrm{H}, H \mathrm{C}=\mathrm{O}), 10.61$ (s, br, $\left.2 \mathrm{H}, \mathrm{NH}\right) .{ }^{13} \mathrm{C} \mathrm{NMR}$ $\left(\mathrm{CDCl}_{3}\right), \delta: 123.7,128.2,129.9,130.4,132.7,132.9,137.9,138.1,141.7\left(\mathrm{C}_{\text {aromatic }}\right), 154.2$ and 155.3 $(C=\mathrm{N}), 191.5(\mathrm{H} C=\mathrm{O})$. DEPT-135 NMR $\left(\mathrm{CDCl}_{3}\right), \delta: 123.7,128.2,129.9,130.4,132.7,132.9$ $\left(C \mathrm{H}_{\text {aromatic }}\right), 191.5(\mathrm{HC}=\mathrm{O})$. Anal. Calcd for $\mathrm{C}_{24} \mathrm{H}_{16} \mathrm{~N}_{5} \mathrm{ClO}_{2} \mathrm{Pt}(636.95)$ : C, 45.26; H, 2.53; N, 11.00 . Found: C, 45.37; H, 2.19; N, 11.25.

\section{$\left[\mathrm{PtCl}\left\{\underline{\mathrm{NH}}=\mathrm{C}\left(p-\mathrm{O}_{2} \mathrm{NC}_{6} \mathrm{H}_{4} \mathrm{CH}_{2}\right) \mathrm{N}=\mathrm{C}\left(\mathrm{C}_{6} \mathrm{H}_{4}\right) \underline{\mathrm{NC}}=\mathrm{NC}\left(p-\mathrm{O}_{2} \mathrm{NC}_{6} \mathrm{H}_{4} \mathrm{CH}_{2}\right)=\underline{\mathrm{NH}}\right\}\right](\mathbf{3 f})$}

Yield: 64\%. IR $\left(\mathrm{cm}^{-1}\right): 3270(\mathrm{NH}), 1605(\mathrm{C}=\mathrm{N}) .{ }^{1} \mathrm{H}$ NMR $\left(\mathrm{CDCl}_{3}\right), \delta: 4.22\left(\mathrm{~s}, 4 \mathrm{H}, \mathrm{CH}_{2}\right) 7.60\left(\mathrm{~d}, J_{\mathrm{HH}} 8.8\right.$ $\mathrm{Hz}, 4 \mathrm{H}, \mathrm{CH}_{\text {aromatic }}$ ), 7.77 (dd, $J_{\mathrm{HH}} 3.1$ and $\left.5.4 \mathrm{~Hz}, 2 \mathrm{H}, \mathrm{CH}_{\text {aromatic }}\right), 8.18$ (dd, $J_{\mathrm{HH}} 3.1$ and $5.4 \mathrm{~Hz}, 2 \mathrm{H}$, 
$\left.\mathrm{CH}_{\text {aromatic }}\right), 8.25\left(\mathrm{~d}, J_{\mathrm{HH}} 8.8 \mathrm{~Hz}, 4 \mathrm{H}, \mathrm{CH}_{\text {aromatic }}\right), 9.96(\mathrm{~s}, \mathrm{br}, 2 \mathrm{H}, \mathrm{NH}) .{ }^{13} \mathrm{C} \mathrm{NMR}\left(\mathrm{CDCl}_{3}\right), \delta: 47.7\left(\mathrm{CH}_{2}\right)$, $123.5,124.1,124.4,128.9,130.4,132.6,134.3,137.6,141.7,147.5\left(\mathrm{C}_{\text {aromatic }}\right), 153.9$ and $159.6(C=\mathrm{N})$. DEPT-135 NMR $\left(\mathrm{CDCl}_{3}\right), \delta$ : $47.7\left(\mathrm{CH}_{2}\right), 123.5,124.1,124.4,128.9,130.4,132.6,134.3\left(C \mathrm{H}_{\text {aromatic }}\right)$. Anal. Calcd for $\mathrm{C}_{24} \mathrm{H}_{18} \mathrm{~N}_{7} \mathrm{ClO}_{4} \mathrm{Pt}$ (698.98): C, 41.24; H, 2.60; N, 14.03. Found: C, 41.55; H, 2.73; N, 14.15 .

2.3. Preparation of the nitrile Pd(II) complexes trans-[PdCl $\left.l_{2}(\mathrm{NCR})_{2}\right] \mathbf{4}(\mathrm{R}=\mathrm{Me}(\mathbf{4 a}), \mathrm{Ph}(\mathbf{4 b}), p$ $\mathrm{MeC}_{6} \mathrm{H}_{4} \mathrm{CH}_{2} \quad(\mathbf{4 c}), \quad p-(\mathrm{HC}=\mathrm{O}) \mathrm{C}_{6} \mathrm{H}_{4} \quad(4 \mathrm{~d}), \quad p-\mathrm{O}_{2} \mathrm{NC}_{6} \mathrm{H}_{4} \mathrm{CH}_{2}$ (4e)) and their reactions with 1,3diiminoisoindoline 2

\subsubsection{Reaction of $\mathrm{PdCl}_{2}$ with liquid nitriles}

$\mathrm{PdCl}_{2}$ (200 mg, $1.127 \mathrm{mmol}$ ) was added at room temperature to acetonitrile, benzonitrile or $p$ tolylacetonitrile $(5 \mathrm{~mL})$, and the mixture was heated under stirring at $70{ }^{\circ} \mathrm{C}$ for $8 \mathrm{~h}$. During the course of the reaction, the brown $\mathrm{PdCl}_{2}$ powder dissolved, forming a homogeneous light yellow solution. The reaction mixture was then dried in vacuo, washed with three $5 \mathrm{~mL}$ portions of diethyl ether and dried under air to give yellow solid compounds trans- $\left[\mathrm{PdCl}_{2}(\mathrm{NCMe})_{2}\right] \mathbf{4 a}$, trans- $\left[\mathrm{PdCl}_{2}(\mathrm{NCPh})_{2}\right] \mathbf{4 b}$ or trans$\left[\mathrm{PdCl}_{2}\left(\mathrm{NC}\left(p-\mathrm{MeC}_{6} \mathrm{H}_{4} \mathrm{CH}_{2}\right)\right)_{2}\right] \mathbf{4 c}$, respectively.

The Pd(II) complexes $\mathbf{4 a}, \mathbf{4 b}$ and $\mathbf{4 c}$ were used without further characterization.

\subsubsection{Reaction of $\mathrm{PdCl}_{2}$ with solid nitriles}

$\mathrm{PdCl}_{2}$ (200 mg, $1.127 \mathrm{mmol}$ ) was added at room temperature to $p$-cyanobenzaldehyde (1477 $\mathrm{mg}, 11.27$ mmol) or $p$-nitrophenylacetonitrile $(1825 \mathrm{mg}, 11.27 \mathrm{mmol})$, and the mixture was heated at the melting point of each nitrile $\left(102\right.$ or $115^{\circ} \mathrm{C}$, respectively) in a sealed glass vial for $12 \mathrm{~h}$. Also in this case, during the course of the reaction, the brown $\mathrm{PdCl}_{2}$ powder dissolved in the melted nitrile, forming a homogeneous light yellow solution. The excess of nitrile was then removed by washing the reaction mixture with three $10 \mathrm{~mL}$ portions of chloroform and dried under air to give yellow solid compounds trans- $\left[\mathrm{PdCl}_{2}\left(\mathrm{NC}\left(p-(\mathrm{HC}=\mathrm{O}) \mathrm{C}_{6} \mathrm{H}_{4}\right)\right)_{2}\right] 4 \mathbf{d}$ or trans- $\left[\mathrm{PdCl}_{2}\left(\mathrm{NC}\left(p-\mathrm{O}_{2} \mathrm{NC}_{6} \mathrm{H}_{4} \mathrm{CH}_{2}\right)\right)_{2}\right] 4 \mathbf{e}$, respectively.

The $\mathrm{Pd}(\mathrm{II})$ complexes $\mathbf{4 d}$ and $\mathbf{4 e}$ were used without further characterization.

2.3.3. Reactions of the nitrile Pd(II) complexes trans-[PdCl $\left.2(\mathrm{NCR})_{2}\right] \mathbf{4}(R=\mathrm{Me}(\mathbf{4 a}), \mathrm{Ph}(\mathbf{4 b}), p$ $\left.\mathrm{MeC}_{6} \mathrm{H}_{4} \mathrm{CH}_{2}(\mathbf{4 c})\right)$ with 1,3-diiminoisoindoline 2 
A solution of $\mathbf{4 a}, \mathbf{4 b}$ or $\mathbf{4 c}(0.532 \mathrm{mmol})$ in acetonitrile, benzonitrile or $p$-tolylacetonitrile $(5 \mathrm{~mL})$, respectively, was added at room temperature to 1,3-diiminoisoindoline 2 (77.2 $\mathrm{mg}, 0.532 \mathrm{mmol})$, and the mixture was refluxed for $2 \mathrm{~h}$ whereupon the solvent was removed in vacuo. The crude residue was washed with three $5 \mathrm{~mL}$ portions of diethyl ether and dried under air.

After a careful IR and NMR analyses of each compound, it was observed that only the Pd(II) complex $\left[\mathrm{PdCl}\left\{\underline{\mathrm{NH}}=\mathrm{C}(\mathrm{Me}) \mathrm{N}=\mathrm{C}\left(\mathrm{C}_{6} \mathrm{H}_{4}\right) \underline{\mathrm{NC}}=\mathrm{NC}(\mathrm{Me})=\underline{\mathrm{NH}}\right\}\right](\mathbf{5 a})$ was formed.

$\left[\mathrm{PdCl}\left\{\underline{\mathrm{NH}}=\mathrm{C}(\mathrm{Me}) \mathrm{N}=\mathrm{C}\left(\mathrm{C}_{6} \mathrm{H}_{4}\right) \underline{\mathrm{NC}}=\mathrm{NC}(\mathrm{Me})=\underline{\mathrm{NH}}\right\}\right](\mathbf{5 a})$

Yield: 65\%. IR $\left(\mathrm{cm}^{-1}\right): 3440(\mathrm{NH}), 1639(\mathrm{C}=\mathrm{N}) .{ }^{1} \mathrm{H}$ NMR (DMSO-d $), \delta: 2.57\left(\mathrm{~s}, 6 \mathrm{H}, \mathrm{CH}_{3}\right), 7.75(\mathrm{~m}$, $\left.2 \mathrm{H}, \mathrm{C} H_{\text {aromatic }}\right), 8.20\left(\mathrm{~m}, 2 \mathrm{H}, \mathrm{C} H_{\text {aromatic }}\right), 10.20(\mathrm{~s}, \mathrm{br}, 1 \mathrm{H}, \mathrm{N} H), 10.33(\mathrm{~s}, \mathrm{br}, 1 \mathrm{H}, \mathrm{N} H) .{ }^{13} \mathrm{C}$ NMR (DMSO- $\left.d_{6}\right), \delta: 27.1\left(\mathrm{CH}_{3}\right), 123.3,124.1,133.8,133.9,134.8,137.9\left(C_{\text {aromatic }}\right), 156.3,164.8(C=\mathrm{N})$.

Anal. Calcd for $\mathrm{C}_{12} \mathrm{H}_{12} \mathrm{~N}_{5} \mathrm{ClPd}$ (368.13): C, 39.15; H, 3.29; N, 19.02. Found: C, 39.46; H, 3.53; N, 19.27 .

2.3.4. Reactions of the nitrile Pd(II) complexes trans-[PdCl $\left.l_{2}(\mathrm{NCR})_{2}\right] \mathbf{4}\left(\mathrm{R}=\mathrm{p}-(\mathrm{HC}=\mathrm{O}) \mathrm{C}_{6} \mathrm{H}_{4}(\mathbf{4 d}), p-\right.$ $\left.\mathrm{O}_{2} \mathrm{NC}_{6} \mathrm{H}_{4} \mathrm{CH}_{2}(4 \mathrm{e})\right)$ with 1,3-diiminoisoindoline 2

A solution of $4 \mathbf{d}$ or $4 \mathbf{e}(0.532 \mathrm{mmol})$ in acetone $(5 \mathrm{~mL})$ was added at room temperature to 1,3diiminoisoindoline $2(77.2 \mathrm{mg}, 0.532 \mathrm{mmol})$, and the mixture was refluxed for $2 \mathrm{~h}$ whereupon the solvent was removed in vacuo. The crude residue was washed with three $5 \mathrm{~mL}$ portions of diethyl ether and dried under air.

After a careful IR and NMR analyses of each reaction product, it was observed the absence of the products of the addition of 1,3-diiminoisoindoline to nitrile group $(\mathrm{N} \equiv C)$, the resulting mixtures contain a number of unidentified products. Additionally, the reaction of $\mathbf{4 e}$ with $\mathbf{2}$ affords also $p$ nitrophenylacetonitrile (resulting from the decomposition of complex 4e).

\subsection{X-ray structure determinations}

For the X-ray structure determination; X-ray-quality single crystal of $\mathrm{Pt}(\mathrm{II})$ complex $\left[\operatorname{PtCl}\left\{\underline{\mathrm{NH}}=\mathrm{C}(\mathrm{Me}) \mathrm{N}=\mathrm{C}\left(\mathrm{C}_{6} \mathrm{H}_{4}\right) \underline{\mathrm{NC}}=\mathrm{NC}(\mathrm{Me})=\underline{\mathrm{NH}}\right\}\right] \quad \mathbf{3 a}$ was obtained by slow evaporation from chloroform. The crystals of 3a were immersed in cryo-oil, mounted in a MiTeGen loop and measured at $120 \mathrm{~K}$. The X-ray diffraction data were collected on a Bruker KappaApex II or an Agilent Technologies 


\section{ACCEPTED MANUSCRIPT}

Supernova using Mo K $\alpha$ radiation $(\lambda=0.70173 \AA)$. The CrysAlisPro [17] program packages were used for cell refinements and data reductions. The structure was solved by charge flipping method using the SUPERFLIP [18] program. A multi-scan absorption correction based on equivalent reflections (CrysAlisPro) was applied to the data. Structural refinement was carried out using SHELXL2014 [19]. The N-H hydrogen atom (H1) was located from the difference Fourier map and refined isotropically. Other hydrogen atoms were positioned geometrically and constrained to ride on their parent atoms, with $\mathrm{C}---\mathrm{H}=0.95-0.98 \AA$ and $\mathrm{U}_{\text {iso }}=1.2-1.5 \mathrm{U}_{\text {eq }}$ (parent atom).

The crystallographic details are summarized in Table 1. Detailed structural parameters are given in supplementary material. CCDC number 1518110 contains the supplementary crystallographic data for this paper. These data can be obtained free of charge from The Cambridge Crystallographic Data Centre via www.ccdc.cam.ac.uk/data_request/cif.

\section{Results and discussion}

\subsection{Reactions of bis(nitrile) Pt(II) complexes 1 with 1,3-diiminoisoindoline 2}

We have recently found [15] that 1,3-diiminoisoindoline, which represents a family of aromatic diimines, exhibits nucleophilic properties toward Pt(II)-bound nitriles (propionitrile and benzonitrile). The stability of the 1,3-diiminoisoindoline and of the products of its addition to nitrile ligands encouraged us to expand this type of reaction to other $\operatorname{Pt}(\mathrm{II})$ and $\mathrm{Pd}(\mathrm{II})$-bound nitriles. 1,3Diiminoisoindoline bears two nucleophilic $s p^{2}$-nitrogen centres and one endocyclic amine group, which upon deprotonation might coordinate to Pt(II) or Pd(II) metal centre forming stable chelated fused metallacycles (Figure 1). 


\section{ACCEPTED MANUSCRIPT}

Auxiliary amine

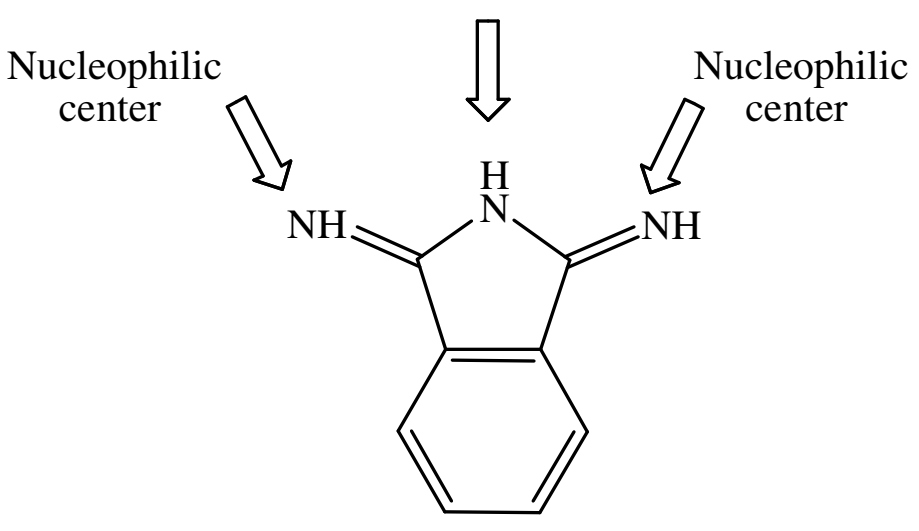

1,3-diiminoisoindoline

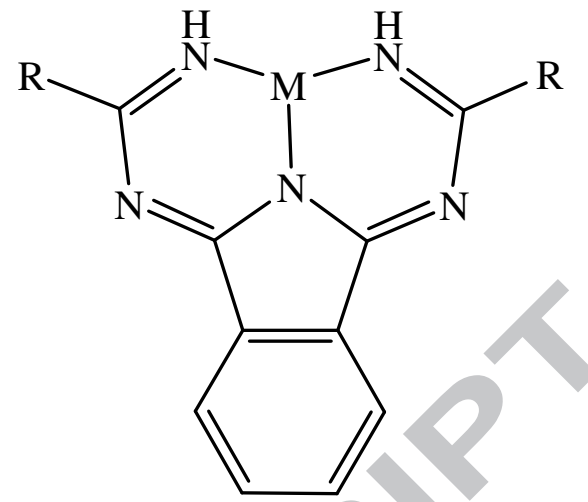

Symmetrical 1,3,5,7,9-pentaazanona1,3,6,8-tetraenato complex

$$
\begin{aligned}
& \mathrm{M}=\mathrm{Pt}, \mathrm{Pd} \\
& \mathrm{R}=\text { alkyl, aryl }
\end{aligned}
$$

Figure 1

Herein, we describe the selective synthesis of new symmetrical (1,3,5,7,9-pentaazanona-1,3,6,8tetraenato)Pt(II) complexes by using various aliphatic and aromatic nitriles as starting bis(nitrile) $\mathrm{Pt}(\mathrm{II})$ complexes and 1,3-diiminoisoindoline as the reacting $s p^{2}$-nitrogen nucleophile.

The starting $\mathrm{Pt}(\mathrm{II})$-bound nitrile complexes trans-[PtCl $\left.2(\mathrm{NCR})_{2}\right] 1(\mathrm{R}=\mathrm{Me}(\mathbf{1 a}), \mathrm{Et}(\mathbf{1 b}), o$ $\mathrm{ClC}_{6} \mathrm{H}_{4}(\mathbf{1 c}), p-\mathrm{ClC}_{6} \mathrm{H}_{4}(\mathbf{1 d}), p-(\mathrm{HC}=\mathrm{O}) \mathrm{C}_{6} \mathrm{H}_{4}(\mathbf{1 e}), p-\mathrm{O}_{2} \mathrm{NC}_{6} \mathrm{H}_{4} \mathrm{CH}_{2}$ (1) ) were prepared, in excellent yields ( $c$ a. 90\%), by reaction of $\mathrm{PtCl}_{2}$ with the respective nitrile under heating. Treatment of complexes 1a-f with one equivalent of 1,3-diiminoisoindoline $\mathrm{HN}=\underline{\mathrm{CC}}_{6} \mathrm{H}_{4} \mathrm{C}(\underline{\mathrm{NH}})=\mathrm{NH} 2$, in refluxing chloroform for $2 \mathrm{~h}$, gives access to the corresponding symmetrical (1,3,5,7,9-pentaazanona-1,3,6,8-tetraenato)Pt(II) complexes $\left[\mathrm{PtCl}\left\{\underline{\mathrm{NH}}=\mathrm{C}(\mathrm{R}) \mathrm{N}=\mathrm{C}\left(\mathrm{C}_{6} \mathrm{H}_{4}\right) \underline{\mathrm{NC}}=\mathrm{NC}(\mathrm{R})=\underline{\mathrm{NH}}\right\}\right]$ 3a-f in good yields $(65-70 \%)$ (Scheme 1). 


\section{ACCEPTED MANUSCRIPT}
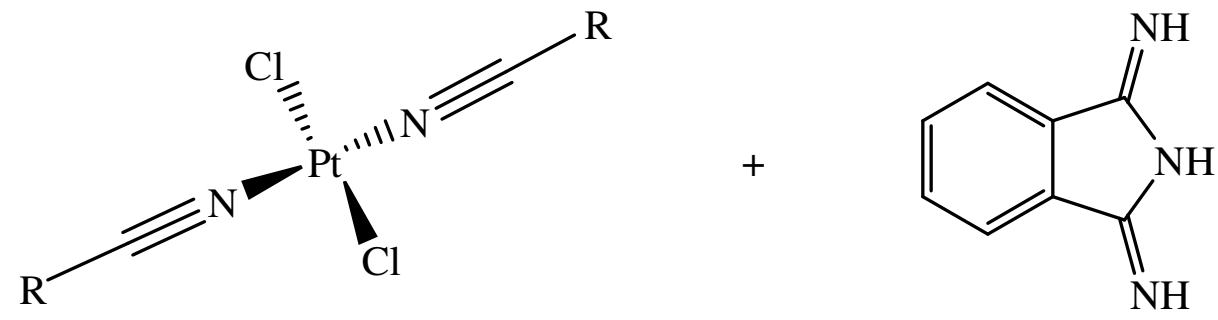

1a: $\mathrm{R}=\mathrm{Me}$

1b: $\mathrm{R}=\mathrm{Et}$

1c: $\mathrm{R}=o-\mathrm{ClC}_{6} \mathrm{H}_{4}$

$\mathrm{CHCl}_{3}$, reflux, $2 \mathrm{~h}$

1d: $\mathrm{R}=p-\mathrm{ClC}_{6} \mathrm{H}_{4}$

1e: $\mathrm{R}=p-(\mathrm{HC}=\mathrm{O}) \mathrm{C}_{6} \mathrm{H}_{4}$

1f: $\mathrm{R}=p-\mathrm{O}_{2} \mathrm{NC}_{6} \mathrm{H}_{4} \mathrm{CH}_{2}$
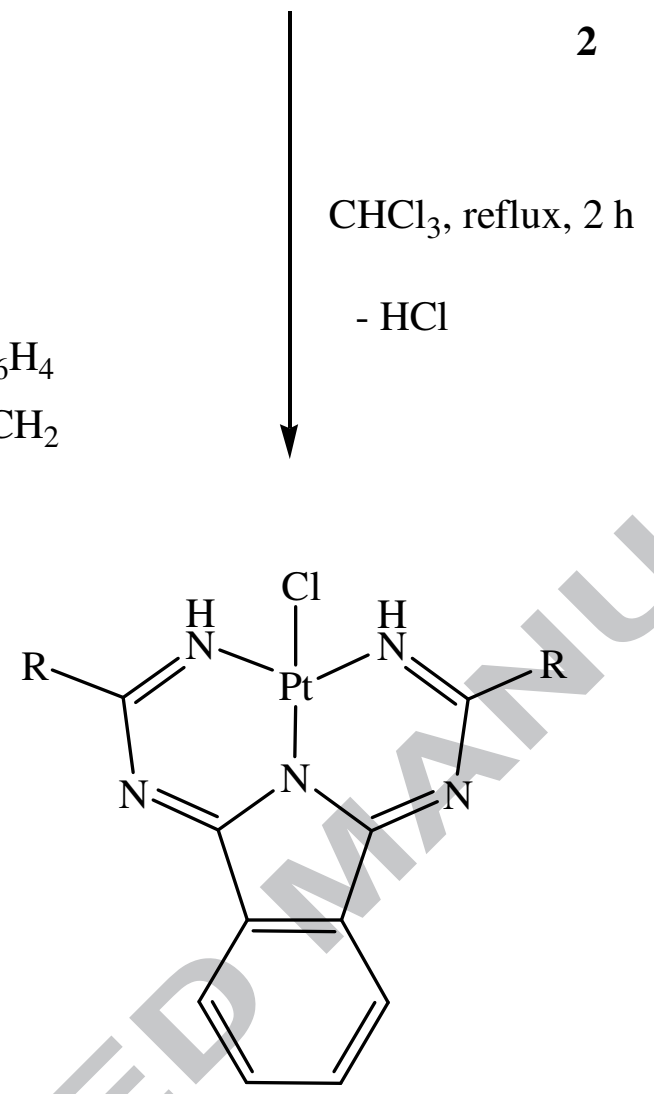

3a: $\mathrm{R}=\mathrm{Me}$

3b: $\mathrm{R}=\mathrm{Et}$

3c: $\mathrm{R}=o-\mathrm{ClC}_{6} \mathrm{H}_{4}$

3d: $\mathrm{R}=p-\mathrm{ClC}_{6} \mathrm{H}_{4}$

3e: $\mathrm{R}=p-(\mathrm{HC}=\mathrm{O}) \mathrm{C}_{6} \mathrm{H}_{4}$

3f: $\mathrm{R}=p-\mathrm{O}_{2} \mathrm{NC}_{6} \mathrm{H}_{4} \mathrm{CH}_{2}$

\section{Scheme 1}

In a blank experiment, a prolonged reflux (24 h) of a mixture of one equivalent of acetonitrile and two equivalents of 1,3-diiminoisoindoline in chloroform showed no addition to cyanocarbon of acetonitrile and only the starting materials were recovered, indicating the $\mathrm{Pt}(\mathrm{II})$-assisted character of the coupling.

The obtained $\mathrm{Pt}(\mathrm{II})$ complexes 3a-f were characterized by $\mathrm{IR},{ }^{1} \mathrm{H},{ }^{13} \mathrm{C}$ and DEPT-135 NMR spectroscopies, elemental analyses and also by X-ray diffraction (in the case of 3a). The IR spectra of complexes 3a-f do not exhibit the typical $v(\mathrm{~N} \equiv \mathrm{C})$ values $\left(2350-2300 \mathrm{~cm}^{-1}\right.$ range), while new bands due to $v(\mathrm{NH})$ and $v(\mathrm{~N}=\mathrm{C})$ are observed at $c a .3255$ and $c a .1648 \mathrm{~cm}^{-1}$, respectively. For example, in the ${ }^{1} \mathrm{H}$ NMR spectrum of the complex $\left[\operatorname{PtCl}\left\{\underline{\mathrm{NH}}=\mathrm{C}(\mathrm{Me}) \mathrm{N}=\mathrm{C}\left(\mathrm{C}_{6} \mathrm{H}_{4}\right) \underline{\mathrm{NC}}=\mathrm{NC}(\mathrm{Me})=\underline{\mathrm{NH}}\right\}\right]$ 3a, the signal of the 


\section{ACCEPTED MANUSCRIPT}

two methyl groups appears as a singlet at $\delta 2.55$, and the $\mathrm{N} H$ protons are exhibited at $\delta 9.80$. The ${ }^{13} \mathrm{C}$ NMR spectrum of 3a shows the characteristic signals of the imine $\mathrm{N}=C$ groups at $\delta 153.1$ and 159.8, and the absence of the nitrile $\mathrm{N} \equiv C$ resonance at $c a$. 116 ppm confirms that the nucleophilic addition of 1,3-diiminoisoindoline 2 occurs to both acetonitrile ligands in 1a (see Experimental Section for more details).

\subsection{X-ray structure crystallography}

Complex 3a has a relatively simple molecular structure. The crystal data and structure refinement are given in Table 1, representative diagram is given in Figure 2 and selected bond distances and angles are provided in Table 2. Other relevant features of the structure of 3a are given as Supplementary Material. The geometry around the Pt ion in 3a is of square planar type, comprising two fused six-membered metallacycles. Atoms C11, Pt1, and N3 are placed on a two-fold rotation axis. Practically planar molecules are forming stacks with metal- $\pi$ and $\pi-\pi$ interactions along the crystallographic c-axis (Figure 3). The shortest atom-atom distances between the adjacent planes are

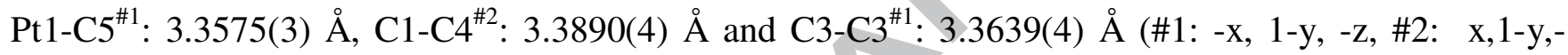
$1 / 2+\mathrm{z}$ ). The stacks are further supported by weak $\mathrm{H}$-bonds between the $\mathrm{NH}$ group and $\mathrm{Cl}$ as well as $\mathrm{CH}_{3}$ group and $\mathrm{Cl}$ (Figure 3). In the previously published structure $\mathbf{3 b}$, the metal compounds are also stacked with shortest C-C distances ranging from $3.344 \AA$ to $3.369 \AA$. In this structure the stacks are supported both by weak $\mathrm{CH}_{2}-\mathrm{Cl} \mathrm{H}$-bonds and much stronger $\mathrm{H}$-bonds involving water of crystallization [15]. 


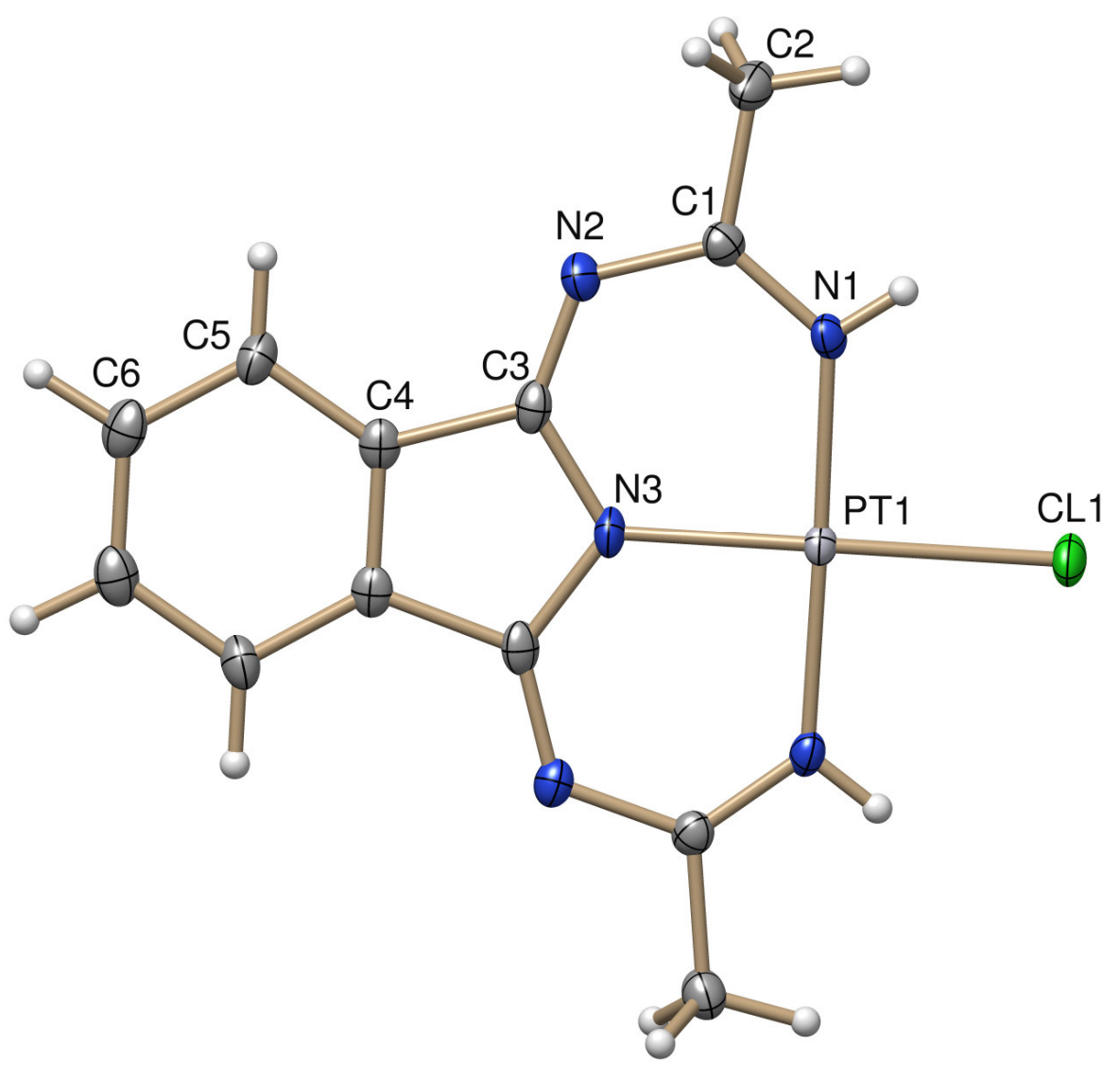

Figure 2. Crystal structure of complex 3a.
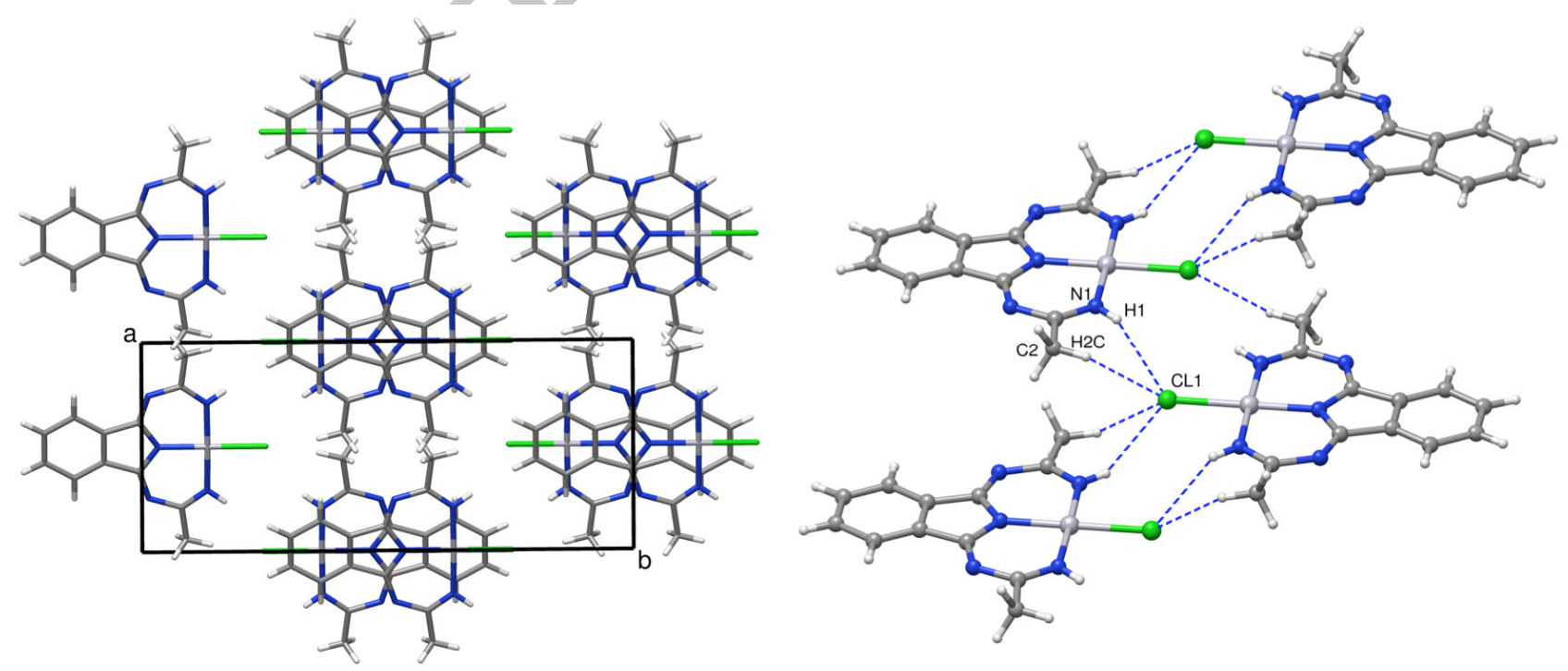

Figure 3. Left: Packing of 3a along the crystallographic c-axis. Right: H-Bonds in 3a. N1-H1: 0.83(9)

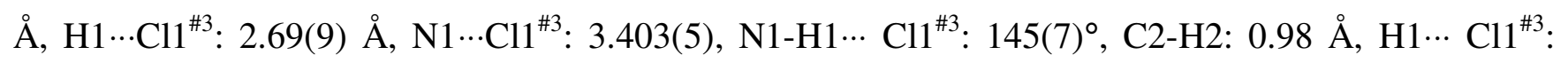
$2.87, \mathrm{C} 2 \cdots \mathrm{Cl1}^{\# 3}: 3.643 \AA$, $\mathrm{C} 2-\mathrm{H} 2 \cdots \mathrm{C} 11^{\# 3}: 137^{\circ}(\# 3:-\mathrm{x}+1 / 2,-\mathrm{y}+1 / 2,-\mathrm{z}+1)$. 
Table 1. Crystal data and structure refinement for complex 3a.

\begin{tabular}{|c|c|}
\hline & $3^{\mathbf{a}}$ \\
\hline empirical formula & $\mathrm{C}_{13} \mathrm{H}_{12} \mathrm{ClN}_{6} \mathrm{Pt}$ \\
\hline $\mathrm{Fw}$ & 456.81 \\
\hline temp $(\mathrm{K})$ & $120(2)$ \\
\hline$\lambda(\AA ̊)$ & 1.54184 \\
\hline cryst syst & Monoclinic \\
\hline space group & $\mathrm{C} 2 / \mathrm{c}$ \\
\hline$a(\AA)$ & $11.8678(5)$ \\
\hline$b(\AA)$ & $19.6347(2)$ \\
\hline$c(\AA)$ & $7.7596(3)$ \\
\hline$\beta\left(^{\circ}\right)$ & $135.018(7)$ \\
\hline$V\left(\AA^{3}\right)$ & $1278.13(14)$ \\
\hline Z & 4 \\
\hline$\rho_{\text {calc }}\left(\mathrm{Mg} / \mathrm{m}^{3}\right)$ & 2.374 \\
\hline$\mu(\mathrm{K} \alpha)\left(\mathrm{mm}^{-1}\right)$ & 22.405 \\
\hline No. reflns. & 13958 \\
\hline Unique reflns. & 1356 \\
\hline GOOF $\left(F^{2}\right)$ & 1.139 \\
\hline$R_{\text {int }}$ & 0.0747 \\
\hline $\mathrm{R}^{\mathrm{a}}(I \geq 2 \sigma)$ & 0.0313 \\
\hline$W R 2^{b}(I \geq 2 \sigma)$ & 0.0823 \\
\hline
\end{tabular}

Table 2. Selected bond lengths $[\AA]$ and angles $\left[{ }^{\circ}\right]$ for complex $\mathbf{3 a}$.

\begin{tabular}{lc}
\hline Pt1-N(3) & $1.961(6)$ \\
Pt1-N(1)\#1 & $1.972(5)$ \\
Pt1-N(1) & $1.972(5)$ \\
Pt1-Cl1 & $2.3178(16)$ \\
& \\
N(3)-Pt1-N(1) & $89.34(11)$ \\
N(1)\#1-Pt1-N(1) & $178.7(2)$ \\
N(3)-Pt1-Cl1 & 180.0 \\
N(1)-Pt1-Cl1 & $90.66(11)$ \\
& \\
\hline
\end{tabular}

Symmetry transformations used to generate equivalent atoms: \#1 -x,y,-z+1/2. 


\section{ACCEPTED MANUSCRIPT}

\section{3. $\quad$ Reactions of bis(nitrile) Pd(II) complexes 4 with 1,3-diiminoisoindoline 2}

Here, we report the selective synthesis of new symmetrical (1,3,5,7,9-pentaazanona-1,3,6,8tetraenato) $\mathrm{Pd}(\mathrm{II})$ complex by using trans-[ $\left.\mathrm{PdCl}_{2}(\mathrm{NCMe})_{2}\right]$ as the starting bis(nitrile) $\mathrm{Pd}(\mathrm{II})$ complex and 1,3-diiminoisoindoline as the reacting $s p^{2}$-nitrogen nucleophile.

The starting $\mathrm{Pd}(\mathrm{II})$-bound acetonitrile complex trans- $\left[\mathrm{PdCl}_{2}(\mathrm{NCMe})_{2}\right]$ 4a was prepared, in excellent yield (89\%), by reaction of $\mathrm{PdCl}_{2}$ with acetonitrile under heating. Treatment of complex $4 \mathbf{a}$ with one equivalent of 1,3-diiminoisoindoline 2 , in refluxing acetonitrile for $2 \mathrm{~h}$, gives access to the symmetrical (1,3,5,7,9-pentaazanona-1,3,6,8-tetraenato) $\mathrm{Pd}(\mathrm{II})$ complex $\left[\mathrm{PdCl}\left\{\underline{\mathrm{NH}}=\mathrm{C}(\mathrm{Me}) \mathrm{N}=\mathrm{C}\left(\mathrm{C}_{6} \mathrm{H}_{4}\right) \underline{\mathrm{NC}}=\mathrm{NC}(\mathrm{Me})=\underline{\mathrm{NH}}\right\}\right] \mathbf{5 a}$ in good yield $(65 \%)$ (Scheme 2, reaction $a$ ).

The IR spectrum of complex 5a do not exhibit the typical $v(\mathrm{~N} \equiv \mathrm{C})$ values $\left(2350-2300 \mathrm{~cm}^{-1}\right.$ range), while new bands due to $v(\mathrm{NH})$ and $v(\mathrm{~N}=\mathrm{C})$ are detected at 3440 and $1639 \mathrm{~cm}^{-1}$, respectively. In the ${ }^{1} \mathrm{H}$ NMR spectrum of $\mathbf{5 a}$, the signal of the two methyl groups appears as a singlet at $\delta 2.57$, and the $\mathrm{N} H$ protons are exhibited at $\delta 10.20$ and 10.33 . The ${ }^{13} \mathrm{C}$ NMR spectrum shows the characteristic signals of the imine $\mathrm{N}=C$ groups at $\delta 156.3$ and 164.8 , and the absence of the nitrile $\mathrm{N} \equiv C$ resonance at $c a .116$ ppm confirms that the nucleophilic addition of 1,3-diiminoisoindoline 2 occurs to both acetonitrile ligands in $\mathbf{4 a}$.

We have also studied the reaction of 1,3-diiminoisoindoline with other bis(nitrile) complexes trans- $\left[\mathrm{PdCl}_{2}(\mathrm{NCR})_{2}\right]$. Hence, the careful NMR analysis of the products of the reaction of trans$\left[\mathrm{PdCl}_{2}(\mathrm{NCR})_{2}\right] 4\left(\mathrm{R}=\mathrm{Ph}(\mathbf{4 b}), p-\mathrm{MeC}_{6} \mathrm{H}_{4} \mathrm{CH}_{2}(\mathbf{4 c}), p-(\mathrm{HC}=\mathrm{O}) \mathrm{C}_{6} \mathrm{H}_{4}(\mathbf{4 d}), p-\mathrm{O}_{2} \mathrm{NC}_{6} \mathrm{H}_{4} \mathrm{CH}_{2}\right.$ (4e)) with 2 shows the formation of a number of unidentified products (in the case of $\mathbf{4 b}$ and $\mathbf{4 d}$ ), or the free nitriles $p$-tolylacetonitrile and $p$-nitrophenylacetonitrile (resulting from the decomposition of the complexes $\mathbf{4 c}$ and $\mathbf{4 e}$, respectively) together with a number of uncharacterized products (Scheme 2, reaction $b$ ). The ${ }^{13} \mathrm{C}$ NMR spectra show the absence of the characteristic signals of the imine $\mathrm{N}=C$ groups at $\delta 153$ and 165. 


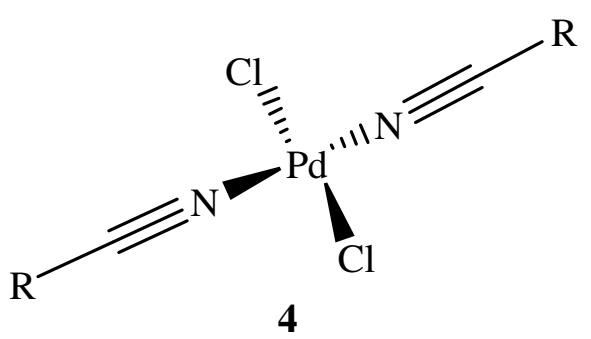

$+$<smiles>N=C1NC(=N)c2ccccc21</smiles>

2 4a: $\mathrm{R}=\mathrm{Me}$

acetonitrile, reflux, $2 \mathrm{~h}$

- $\mathrm{HCl}$

(a)

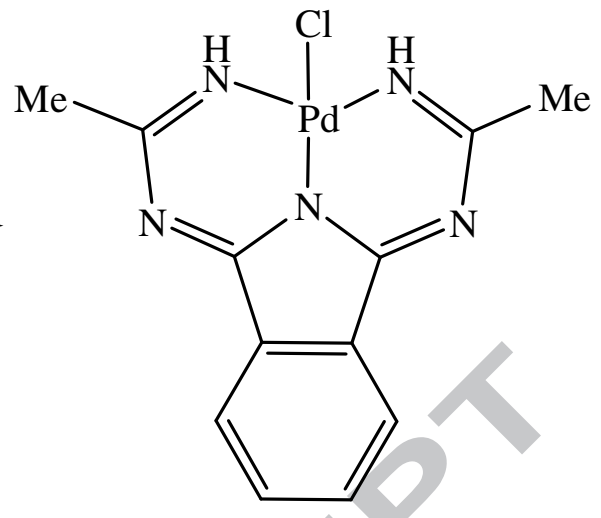

$5 a$

4b: $\mathrm{R}=\mathrm{Ph}$

4c: $\mathrm{R}=p-\mathrm{MeC}_{6} \mathrm{H}_{4} \mathrm{CH}_{2}$

4d: $\mathrm{R}=p-(\mathrm{HC}=\mathrm{O}) \mathrm{C}_{6} \mathrm{H}_{4}$

4e: $\mathrm{R}=p-\mathrm{O}_{2} \mathrm{NC}_{6} \mathrm{H}_{4} \mathrm{CH}_{2}$

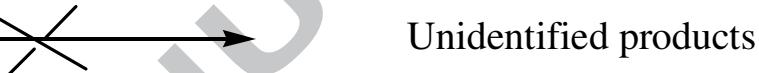

reflux, $2 \mathrm{~h}$

(b)

Scheme 2

\section{4. $U V$-vis absorption spectra}

The absorption spectra of the Pt(II) complexes 3a and $\mathbf{3 b}$ exhibit a band at 400-430 nm typical of a metal-to-ligand charge transfer (MLCT) low energy transition, and intrinsic of the $\mathrm{d}^{8}$ platinum complexes. The bands located around $330 \mathrm{~nm}$ are more ligand-centered and, due to the solvent cut-off, it was not possible to observe the highest energy absorption bands, which should be located below 250 $\mathrm{nm}$ for this type of complexes [14], and correspond to ${ }^{1}\left(\pi-\pi^{\star}\right)$ IL (intraligand) transitions.

The absorption data (maximum absorption wavelengths and corresponding molar absorption coefficients) are summarized in Table 3.

Table 3. Wavelengths of the absorption maxima, respective molar absorption coefficients, luminescence quantum yields and lifetimes for Pt(II) complexes $\mathbf{3 a}$ and $\mathbf{3 b}$.

\begin{tabular}{|l|c|c|c|c|}
\hline & $\begin{array}{c}\lambda_{\max } / \mathrm{nm}\left(\varepsilon / \mathrm{M}^{-1} \mathrm{~cm}^{-1}\right), \\
\mathrm{CH}_{2} \mathrm{Cl}_{2}\end{array}$ & $\lambda_{\mathrm{em}} / \mathrm{nm}, \mathrm{CH}_{2} \mathrm{Cl}_{2}$ & $\Phi_{\mathrm{L}} / \times 10^{-3}, \mathrm{CH}_{2} \mathrm{Cl}_{2}$ & $\tau / \mathrm{ns}$ \\
\hline
\end{tabular}




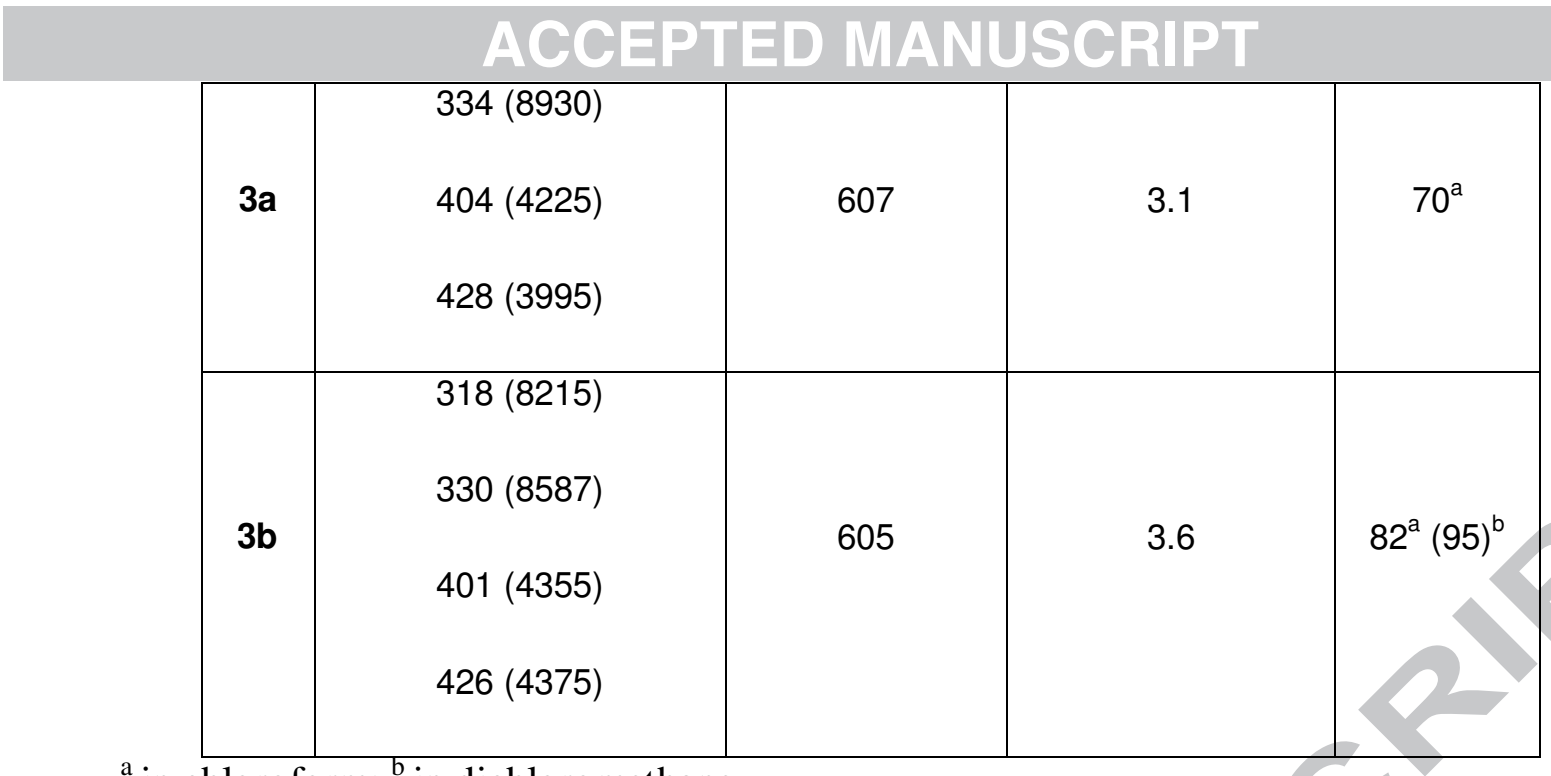

${ }^{a}$ in chloroform; ${ }^{b}$ in dichloromethane.

\subsection{Luminescence emission spectra}

Both compounds are emissive in dichloromethane solution, and exhibit very similar emission spectra, luminescence quantum yields and lifetimes. This might be due to the structural similarity between the two compounds, which only differ by one carbon atom in two of the imine substituents. The emission data are collected in Table 3. Absorption, emission and excitation spectra of both complexes are represented in Figures 4 and 5. 


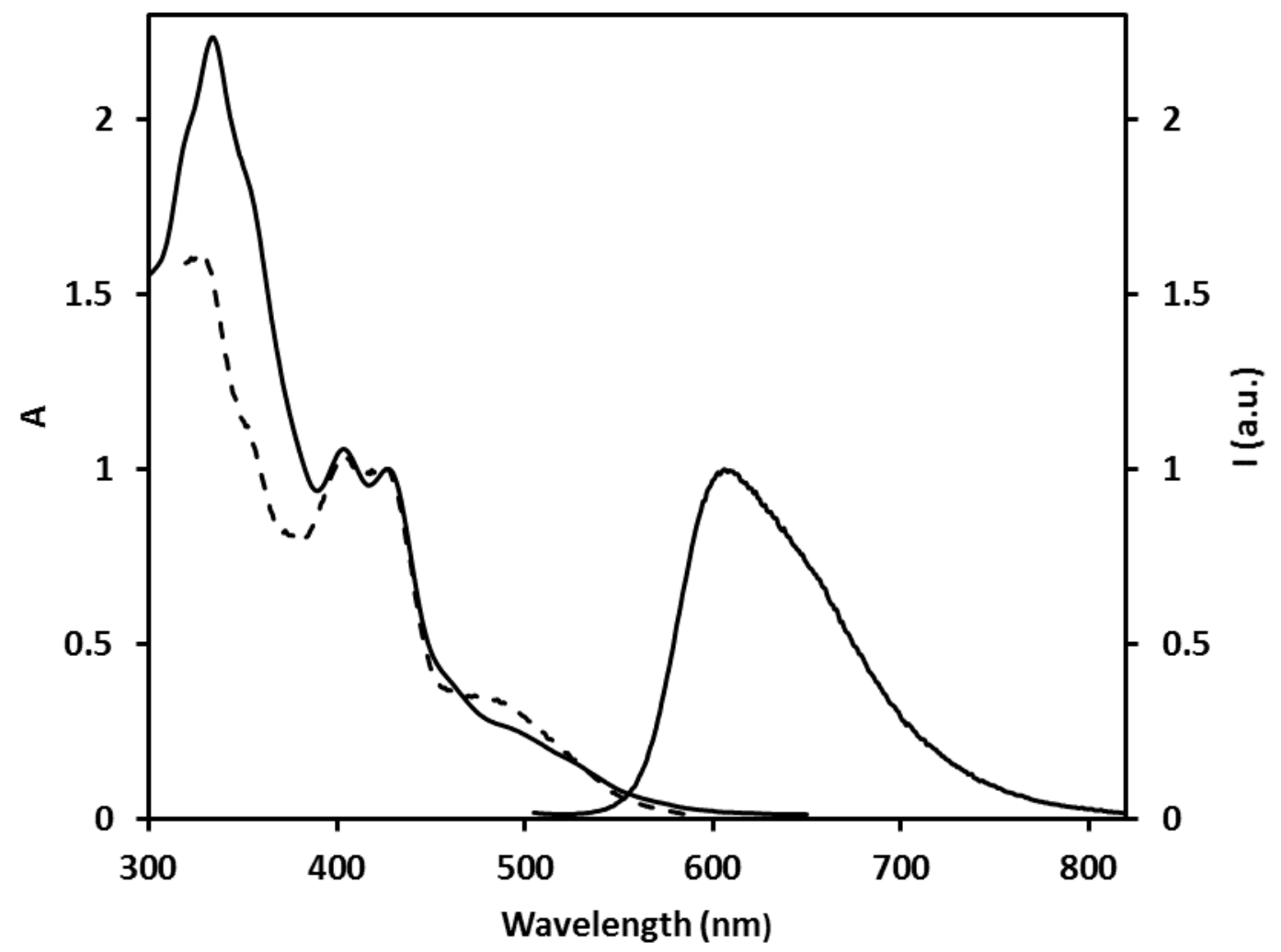

Figure 4. Normalized absorption, emission (solid lines) and excitation (dashed line) spectra of compound 3a in $\mathrm{CH}_{2} \mathrm{Cl}_{2}$. $\lambda_{\text {exc }}=428 \mathrm{~nm}$. 


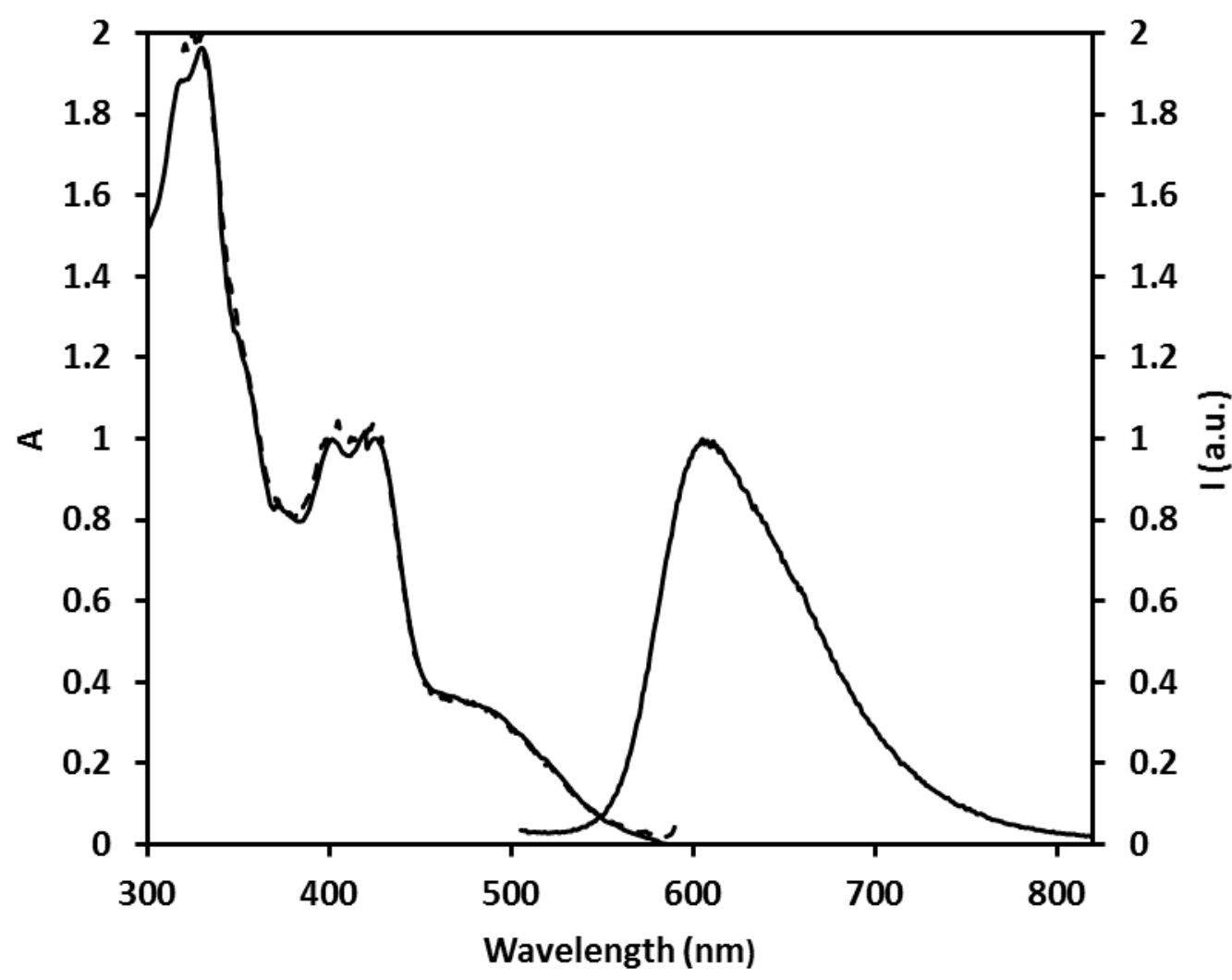

Figure 5. Normalized absorption, emission (solid lines) and excitation (dashed line) spectra of compound $3 \mathbf{b}$ in $\mathrm{CH}_{2} \mathrm{Cl}_{2}$. $\lambda_{\text {exc }}=426 \mathrm{~nm}$.

By comparison of the luminescence lifetimes assessed for these compounds with those collected for $\mathrm{Pt}(\mathrm{II})$ imidoylamidinates [14], it is possible to observe that the former are several orders of magnitude lower. This might be due to the fact that $\mathrm{Pt}(\mathrm{II})$ complexes $\mathbf{3 a}$ and $\mathbf{3 b}$ exhibit a smaller degree of electronic delocalization, which ultimately governs the lifetime of the emissive triplet state. It is worth mentioning that degassing produced no effect on their lifetimes and quantum yields.

3.6.

\section{Solid state luminescence}

Compounds 3a and $\mathbf{3 b}$ also exhibited luminescence in solid state. The characterization was performed following the procedure described in section 2.1, and the resulting emission spectra are depicted in Figure 6. 


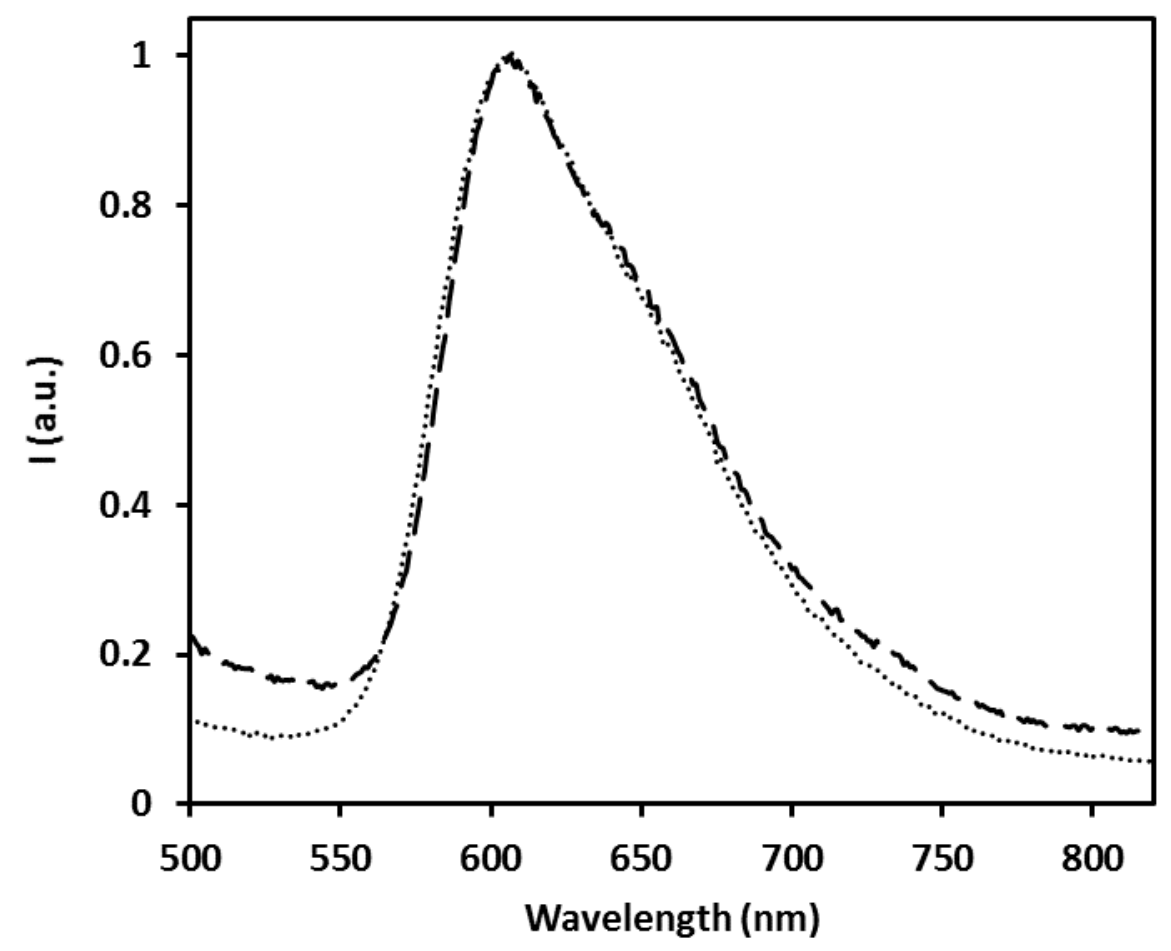

Figure 6. Normalized emission (dashed line) spectra of compounds 3a (dashed line) and 3b (dotted line) in polystyrene film. $\lambda_{\text {exc }}=441 \mathrm{~nm}$.

The spectra are very similar to the ones obtained in solution for both compounds, except for the slight elevation of the baseline, which is common for solid samples, owing to scattering. Regarding luminescence lifetimes, a significant increase occurs in the solid state, a common lifetime of $160 \mathrm{~ns}$ being measured for both $\mathbf{3 a}$ and $\mathbf{3 b}$. Immobilization of the compounds in the solid matrix significantly reduces collisional quenching, hence increasing the excited state lifetime.

\section{Conclusions}

The results of this work can be summarized under four perspectives. First, the reaction between trans-[PtCl $\left.2(\mathrm{NCR})_{2}\right] \mathbf{1}$ and 1,3-diiminoisoindoline $\mathbf{2}$ affords (1,3,5,7,9-pentaazanona-1,3,6,8tetraenato) $\mathrm{Pt}(\mathrm{II})$ complexes 3. Second, we achieved the selective synthesis of new symmetrical (1,3,5,7,9-pentaazanona-1,3,6,8-tetraenato)Pd(II) complex 5a by using trans-[ $\left.\mathrm{PdCl}_{2}(\mathrm{NCMe})_{2}\right] \mathbf{4 a}$ and 1,3-diiminoisoindoline $\mathbf{2}$, and the system represents a novel reactivity mode which has never been reported so far. Third, the developed synthetic methods operate under mild conditions (reflux, $2 \mathrm{~h}$ ), furnish pure symmetrical (1,3,5,7,9-pentaazanona-1,3,6,8-tetraenato) $\mathrm{Pt}(\mathrm{II})$ and $\mathrm{Pd}(\mathrm{II})$ complexes, and, in contrast to the preparation of (1,3,5-triazapentadienato) Ni(II) complexes [7a], does not need the use of a base. Fourth, the emissive Pt(II) complexes 3a and $\mathbf{3 b}$ exhibited significantly lower luminescent 


\section{ACCEPTED MANUSCRIPT}

lifetimes in solution when compared to their $\mathrm{Pt}(\mathrm{II})$ imidoylamidinate analogues [14], which is a consequence of a smaller electronic delocalization in the former. Furthermore, absorption spectra allowed the assignment of the typical ${ }^{3} \mathrm{MLCT}$ transition bands, characteristic of this type of transitionmetal complexes.

\section{Acknowledgements}

This project was funded by the Deanship of Scientific Research (DSR) at King Abdulaziz University, Jeddah, under grant no. (G-100-662-37). The authors, therefore, acknowledge with thanks DSR for technical and financial support. B. P. acknowledges Fundação para a Ciência e a Tecnologia (FCT) for the grant SFRH/BPD/104295/2014.

\section{Appendix A. Supplementary data}

Supplementary data associated with this article (X-ray crystallographic data and copies of ${ }^{1} \mathrm{H},{ }^{13} \mathrm{C}$ and DEPT-135 NMR spectra) can be found, in the online version, at http://dx.doi.org/.....

\section{References}

[1] (a) A.J.L. Pombeiro, V.Yu. Kukushkin, in: Comprehensive Coordination Chemistry II, Elsevier, Amsterdam, $2^{\text {nd }}$ edn., 2004, Vol. 1 (A.B.P. Lever, ed.), Ch.1.34, p. 639;

(b) V.Yu. Kukushkin, A.J.L. Pombeiro, Chem. Rev. 102 (2002) 1771.

[2] (a) J. Lasri, M.F.C. Guedes da Silva, M.A. Januário Charmier, A.J.L. Pombeiro, Eur. J. Inorg. Chem. (2008) 3668;

(b) J. Lasri, M.A. Januário Charmier, M.F.C. Guedes da Silva, A.J.L. Pombeiro, Dalton Trans. (2007) 3259 ;

(c) J. Lasri, M.A. Januário Charmier, M.F.C Guedes da Silva, A.J.L. Pombeiro, Dalton Trans. (2006) 5062.

[3] (a) J. Vicente, J.A. Abad, M.J. López-Sáez, P.G. Jones, Angew. Chem. Int. Ed. 44 (2005) 6001;

(b) J. Vicente, M.T. Chicote, M.A. Beswick, M.C. Ramírez de Arellano, Inorg. Chem. 35 (1996) 6592;

(c) J. Vicente, M.T. Chicote, M.C. Lagunas, P.G. Jones, Inorg. Chem. 34 (1995) 5441;

(d) J. Vicente, M.T. Chicote, J. Fernández-Baeza, F.J. Lahoz, J.A. Lopez, Inorg. Chem. 30 (1991) 3617.

[4] (a) J. Lasri, S.M. Soliman, M.A. Januário Charmier, M. Ríos-Gutiérrez, L.R. Domingo, Polyhedron 98 (2015) 55;

(b) J. Lasri, Polyhedron 57 (2013) 20; 
(c) J. Lasri, M.J. Fernández Rodríguez, M.F.C. Guedes da Silva, P. Smoleński, M.N. Kopylovich, J.J.R. Fraústo da Silva, A.J.L. Pombeiro, J. Organomet. Chem. 696 (2011) 3513;

(d) R.R. Fernandes, J. Lasri, M.F.C. Guedes da Silva, A.M.F. Palavra, J.A.L. da Silva, J.J.R. Fraústo da Silva, A.J.L. Pombeiro, Adv. Synth. Catal. 353 (2011) 1153;

(e) J. Lasri, M.F.C. Guedes da Silva, M.N. Kopylovich, B.G. Mukhopadhyay, A.J.L. Pombeiro, Eur. J. Inorg. Chem. (2009) 5541;

(f) J. Lasri, M.F.C Guedes da Silva, M.N. Kopylovich, S. Mukhopadhyay, M.A. Januário Charmier, A.J.L. Pombeiro, Dalton Trans. (2009) 2210;

(g) S. Mukhopadhyay, B.G. Mukhopadhyay, M.F.C. Guedes da Silva, J. Lasri, M.A. Januário Charmier, A.J.L. Pombeiro, Inorg. Chem. 47 (2008) 11334;

(h) S. Mukhopadhyay, J. Lasri, M.F.C. Guedes da Silva, M.A. Januário Charmier, A.J.L. Pombeiro, Polyhedron. 27 (2008) 2883;

(i) J. Lasri, M.N. Kopylovich, M.F.C. Guedes da Silva, M.A. Januário Charmier, A.J.L. Pombeiro, Chem. Eur. J. 14 (2008) 9312;

(j) S. Mukhopadhyay, J. Lasri, M.A. Januário Charmier, M.F.C. Guedes da Silva, A.J.L. Pombeiro, Dalton Trans. (2007) 5297;

(k) J. Lasri, M.A. Januário Charmier, M. Haukka, A.J.L. Pombeiro, J. Org. Chem. 72 (2007) 750.

[5] (a) M. Bai, P.C. Lo, J. Ye, C. Wu, W.P. Fong, D.K.P. Ng, Org. Biomol. Chem. 9 (2011) 7028;

(b) D.C. Xia, S.K. Yu, R.S. Shen, C.Y. Ma, C.H. Cheng, D.M. Ji, Z.Q. Fan, X. Wang, G.T. Du, Dyes and Pigments 78 (2008) 84;

(c) M.Q. Tian, T. Wada, H. Sasabe, Dyes and Pigments 52 (2002) 1;

(d) T. Schneider, H. Heckmann, M. Barthel, M. Hanack, Eur. J. Org. Chem. (2001) 3055.

[6] O.N. Trukhina, M.S. Rodríguez-Morgade, S. Wolfrum, E. Caballero, N. Snejko, E.A. Danilova, E. Gutierrez-Puebla, M.K. Islyaikin, D.M. Guldi, T. Torres, J. Am. Chem. Soc. 132 (2010) 12991.

[7] (a) P.V. Gushchin, K.V. Luzyanin, M.N. Kopylovich, M. Haukka, A.J.L. Pombeiro, V.Yu. Kukushkin, Inorg. Chem. 47 (2008) 3088;

(b) M.N. Kopylovich, K.V. Luzyanin, M. Haukka, A.J.L. Pombeiro, V.Yu. Kukushkin, Dalton Trans. (2008) 5220;

(c) K.V. Luzyanin, A.J.L. Pombeiro, M. Haukka, V.Yu. Kukushkin, Organometallics 27 (2008) 5379.

[8] N. Heße, R. Frölich, B. Wibbeling, E.-U. Wüthwein, Eur. J. Org. Chem. (2006) 3923.

[9] M.N. Kopylovich, A.J.L. Pombeiro, Coord. Chem. Rev. 255 (2011) 339.

[10] (a) W.J. Bland, R.D.W. Kemmitt, I.W. Nowell, D.R. Russell, Chem. Commun. (1968) 1065; 


\section{ACCEPTED MANUSCRIPT}

(b) M. Bottrill, R. Goddard, M. Green, R.P. Hughes, M.K. Lloyd, S.H. Taylor, P. Woodward, J. Chem. Soc., Dalton Trans. (1975) 1150;

(c) V. Robinson, G.E. Taylor, P. Woodward, M.I. Bruce, R.C. Wallis, J. Chem. Soc., Dalton Trans. (1981) 1169.

[11] M.N. Kopylovich, A.J.L. Pombeiro, A. Fischer, L. Kloo, V.Yu. Kukushkin, Inorg.Chem. 42 (2003) 7239.

[12] (a) M.N. Kopylovich, J. Lasri, M.F.C. Guedes da Silva, A.J.L. Pombeiro, Eur. J. Inorg. Chem. (2011) 377;

(b) M.N. Kopylovich, J. Lasri, M.F.C. Guedes da Silva, A.J.L. Pombeiro, Dalton Trans. (2009) 3074.

[13] (a) M.N. Kopylovich, Y.Yu. Karabach, M.F.C. Guedes da Silva, P.J. Figiel, J. Lasri, A.J.L. Pombeiro, Chem. Eur. J. 18 (2012) 899;

(b) P.J. Figiel, M.N. Kopylovich, J. Lasri, M.F.C. Guedes da Silva, J.J.R. Fraústo da Silva, A.J.L. Pombeiro, Chem Commun. 46 (2010) 2766.

[14] G.H. Sarova, N.A. Bokach, A.A. Fedorov, M.N. Berberan-Santos, V.Yu. Kukushkin, M. Haukka, J.J.R. Fraústo da Silva, A.J.L. Pombeiro, Dalton Trans. (2006) 3798.

[15] J. Lasri, M.L. Kuznetsov, M.F.C. Guedes da Silva, A.J.L. Pombeiro, Inorg. Chem. 51 (2012) 10774.

[16] F. Menezes, A. Fedorov, C. Baleizao, B. Valeur, M.N. Berberan-Santos, Methods Appl. Fluorescence 1 (2013) 015002.

[17] Agilent, CrysAlisPro, Agilent Technologies inc., Yarnton, Oxfordshire, England, 2013.

[18] L. Palatinus, G. Chapuis, J. Appl. Cryst. 40 (2007) 786.

[19] G.M. Sheldrick, Acta Cryst. A64 (2008) 112. 
$\mathrm{Pt}(\mathrm{II})$ and $\mathrm{Pd}(\mathrm{II})$-assisted coupling of nitriles and 1,3-diiminoisoindoline: Synthesis and luminescence properties of (1,3,5,7,9-pentaazanona-1,3,6,8-tetraenato) $\mathrm{Pt}(\mathrm{II})$ and Pd(II) complexes

Jamal Lasri*, Bruno Pedras, Matti Haukka and Mário N. Berberan-Santos

Graphical abstract
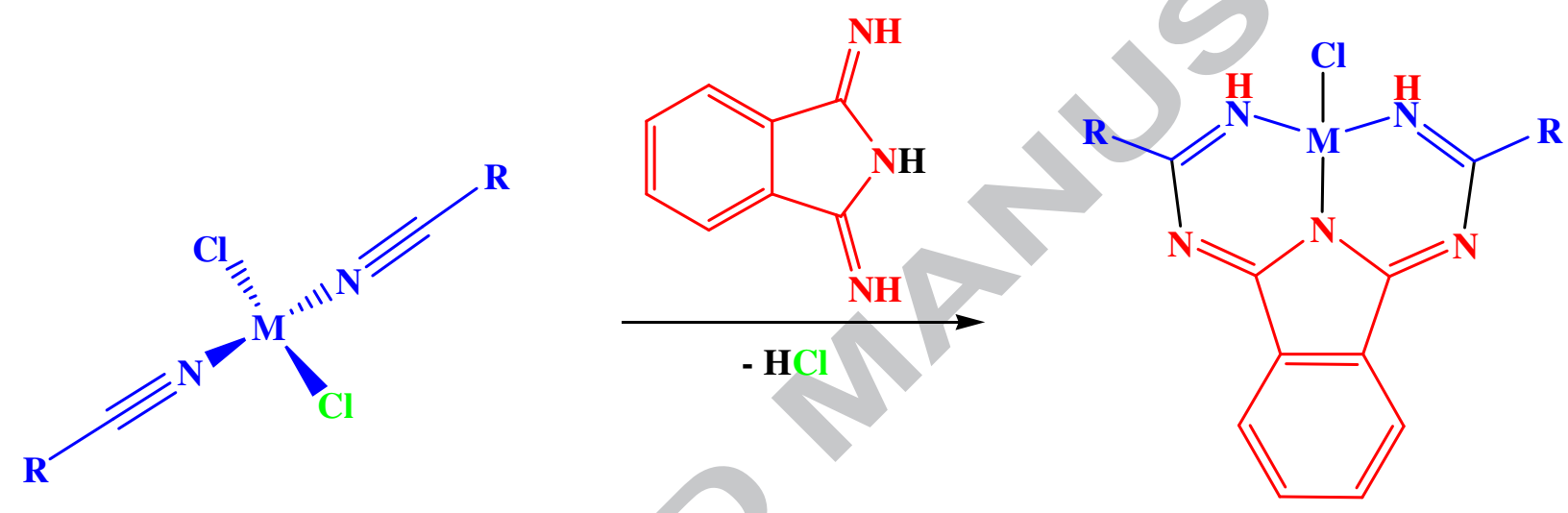

$\mathbf{M}=\mathbf{P t}, \mathbf{P d}$

$\mathrm{Pt}$ (II) and Pd(II)-assisted coupling of nitriles and 1,3-diiminoisoindoline: Synthesis and luminescence properties of (1,3,5,7,9-pentaazanona-1,3,6,8-tetraenato) Pt(II) and Pd(II) complexes

Jamal Lasri*, Bruno Pedras, Matti Haukka and Mário N. Berberan-Santos

Synopsis 
Novel (1,3,5,7,9-pentaazanona-1,3,6,8-tetraenato)Pt(II) and Pd(II) complexes were prepared by nucleophilic additions of 1,3-diiminoisoindoline to $\mathrm{Pt}(\mathrm{II})$ or $\mathrm{Pd}(\mathrm{II})$-bound nitriles, respectively. Photophysical characterization allowed elucidation of the electronic structure of some of the complexes. 